\title{
Stacking-controllable interlayer coupling and symmetric configuration of multilayered $\mathrm{MoS}_{2}$
}

\author{
Sachin M Shinde ${ }^{1,4}$, Krishna P Dhakal ${ }^{1,4}$, Xiang Chen ${ }^{1,4}$, Won Seok Yun ${ }^{2}$, JaeDong Lee ${ }^{2}$, Hyunmin Kim ${ }^{3}$ \\ and Jong-Hyun Ahn ${ }^{1}$
}

The stacking order in layered transition-metal dichalcogenides (TMDCs) induces variations in the electronic and interlayer couplings. Therefore, controlling the stacking orientations when synthesizing TMDCs is desirable but remains a significant challenge. Here, we developed and showed the growth kinetics of different shapes and stacking orders in as-grown multi-stacked $\mathrm{MoS}_{2}$ crystals and revealed the stacking-order-induced interlayer separations, spin-orbit couplings (SOCs), and symmetry variations. Raman spectra in $A A(A . .$.$) -stacked crystals demonstrated blueshifted out-of-plane \left(A_{1 g}\right)$ and in-plane $\left(E_{2 g}{ }^{1}\right)$ phonon frequencies, representing a greater reduction of the van der Waals gap compared to conventional AB(A...)-stacking. Our observations, together with first-principles calculations, revealed distinct excitonic phenomena due to various stacking orientations. As a result, the photoluminescence emission was improved in the AA(A...)-stacking configuration. Additionally, calculations showed that the valence-band maxima (VBM) at the $K$ point of the AA(A...)-stacking configuration was separated into multiple sub-bands, indicating the presence of stronger SOC. We demonstrated that AA(A...)-stacking emitted an intense second-harmonic signal (SHG) as a fingerprint of the more augmented non-centrosymmetric stacking and enabled SOC-induced splitting at the VBM. We further highlighted the superiority of four-wave mixing-correlated SHG microscopy to quickly resolve the symmetries and multi-domain crystalline phases of differently shaped crystals. Our study based on crystals with different shapes and multiple stacking configurations provides a new avenue for development of future optoelectronic devices.

NPG Asia Materials (2018) 10, e468; doi:10.1038/am.2017.226; published online 9 February 2018

\section{INTRODUCTION}

Two-dimensional (2D) transition-metal dichalcogenides, including molybdenum disulfide $\left(\mathrm{MoS}_{2}\right)$, have great potential applications in advanced electronic and optoelectronic devices. ${ }^{1-10}$ Thus, such materials are being intensively studied with a focus on controllable synthesis with excellent optical and electrical performances. Monolayer $\mathrm{MoS}_{2}$ has a direct band-gap structure, exhibiting many intriguing physical properties, such as strong photoluminescence (PL), electronic mobility, and valley and spin polarization. ${ }^{1-3}$ Furthermore, the above optoelectronic properties can be controlled and advanced by adopting a proper design with multiple layered samples with an indirect band gap. In this sense, the successful design of a transition-metal dichalcogenide-based device lies in finding an optimal way to freely control the film thickness and stacking order from a single sheet to multiple layered sheets.

Recently, an atmospheric-pressure chemical vapor deposition (APCVD) system was developed, enabling multi-stacked $\mathrm{MoS}_{2}$ crystals with all possible orientations between $0^{\circ}$ and $60^{\circ}$ to be obtained in a single set of experiments. ${ }^{11}$ In this process, the temperature was identified as a key parameter affecting the growth kinetics of the CVDbased $\mathrm{MoS}_{2}$ synthesis with a positive correlation between the growth rate and film thickness. ${ }^{9-14}$ To date, stacking of up to three layers of $\mathrm{MoS}_{2}$ with various orientations has only been obtained by CVD at high temperatures $\left(\geqslant 850^{\circ} \mathrm{C}\right) .{ }^{11-14}$ In conventional CVD synthesis, high-order stacking is only possible at high temperatures. In contrast, in this research, our approach involves growing $\mathrm{MoS}_{2}$ stacked crystals with high stacking orders (AAA and $\mathrm{ABA}$ ) at the lowest possible temperature. One way to achieve a lower growth temperature is the addition of alkali halides to $\mathrm{MoO}_{3}{ }^{15}$ This addition of $\mathrm{NaCl}$ results in the formation of low sublimating Mo-oxychlorides, which in turn are responsible for the growth of highly stacked $\mathrm{MoS}_{2}$ crystals.

Stacking of $\mathrm{MoS}_{2}$ crystals can be categorized in three groups: (a) repeated-layer piling with the same orientation, as in $\mathrm{AA}(\mathrm{A} \ldots)$; (b) repeated-layer piling with alternating orientations, as in $\mathrm{AB}(\mathrm{AB} \ldots)$; and (c) a mixed-layer piling with false order in the abovementioned stacking orders, such as $\mathrm{AAB} \ldots$ or $\mathrm{AABB} \ldots{ }^{11}$ Intensive scanning transmission electron microscopy analysis has proven the existence of an $\mathrm{AA}(\mathrm{A} . .$.$) -type arrangement in 3R-phase crystals possessing broken$

\footnotetext{
${ }^{1}$ School of Electrical and Electronic Engineering, Yonsei University, Seoul, Republic of Korea; ${ }^{2}$ Department of Emerging Materials Science, DGIST, Daegu, Republic of Korea and ${ }^{3}$ Companion Diagnostics \& Medical Technology Convergence Research Lab, DGIST, Daegu, Republic of Korea

${ }^{4}$ These authors contributed equally to this work.

Correspondence: Dr H Kim, Companion Diagnostics \& Medical Technology Research Group, DGIST, Daegu 42988, Republic of Korea.

E-mail: hyunmin.kim@dgist.ac.kr or

Professor J-H Ahn, School of Electrical \& Electronic Engineering, Yonsei University, 50 Yonsei-ro, Seodaemoon-gu, Seoul 03722, Republic of Korea.

E-mail: ahnj@yonsei.ac.kr

Received 7 September 2017; revised 5 November 2017; accepted 6 November 2017
} 
inversion symmetry, whereas $\mathrm{AB}(\mathrm{A} \ldots)$-type stacking can form a $2 \mathrm{H}$-phase similar to that of natural crystals, where the net symmetry of the inversion depends whether there is an odd or even number of layers. ${ }^{10-13,16,17}$ Moreover, the remaining stacking order, such as A $(\mathrm{BB} \ldots)$ or $\mathrm{AA}(\mathrm{BA} \ldots)$, with a false order of either $\mathrm{AB}(\mathrm{A} \ldots)$ or $\mathrm{AA}(\mathrm{A} \ldots)$ gives rise to a combination of $2 \mathrm{H}$ and $3 \mathrm{R}$-phases. Note that these stacking orientations tune the electronic properties by engineering interlayer distances and structural symmetry. ${ }^{3,11,13}$ In particular, the spin-orbit coupling (SOC) effect, valley polarization, and representative nonlinear optical properties, such as second-harmonic generation (SHG), are highly affected by the stacking configuration and subsequent structural symmetry. ${ }^{3-5,11,16,17}$ To the best of our knowledge, few studies of stacking-orientation-controlled samples of more than three layers and subsequent characterization with PL and Raman spectroscopy have been conducted. ${ }^{10-13,18}$ Furthermore, studying the nonlinear optical properties, such as SHG or four-wave mixing (FWM), as a function of the layer number and stacking orientation of the multi-stacked crystals grown by CVD have scarcely been sought. ${ }^{4-6}$ The minute variations of the band structures according to the geometry and order of stacking are sufficient to modulate the nonlinear optical susceptibility, and hence, versatile $\mathrm{MoS}_{2}$ crystals synthesized by CVD play a crucial role in understanding fundamental optoelectronic properties.

In this report, we provide a synthetic modality of multi-stacked $\mathrm{MoS}_{2}$ crystals with different orientations and characterize their optical properties. More than 18 definable layer thicknesses are successfully synthesized using monolayer and bilayer $\mathrm{MoS}_{2}$ crystals as seeds layers. We further explore the PL, Raman, and absorption spectroscopies of various stacking-oriented samples and observe meaningful gradual spectroscopic evolutions induced by structural changes and interlayer interactions. The first-principles calculations are well consistent with the observed electronic band structures and reveal the excitonic emission. Additionally, the degree of the SOC effect in splitting the valence-band maxima at the $\mathrm{K}$ point is modulated by the stacking orientation. Additionally, the centers of symmetry, stacking orientations, and crystalline domains in a single specimen are more deeply assessed using FWM and SHG. The SHG signal of the AA(A...)stacking crystal is drastically enhanced compared to the naturally occurring $2 \mathrm{H}$-type $\mathrm{AB}(\mathrm{A} . .$.$) -stacking and increases gradually with an$ increasing layer thickness. In contrast to $\mathrm{AA}(\mathrm{A} \ldots)$-stacking, $\mathrm{AB}(\mathrm{A} \ldots)$ stacking has the strongest SHG signal for a monolayer and then decreases gradually for an odd number of layers with no measurable signal for an even number of layers. Our approach of synthesizing high-quality multi-stacked $\mathrm{MoS}_{2}$ crystals and the associated background understanding provides a valuable direction for the future design of optoelectronic devices using diversely stacked few-layer $\mathrm{MoS}_{2}$ flakes.

\section{MATERIALS AND METHODS}

\section{CVD synthesis of multi-stacked $\mathrm{MoS}_{2}$}

The $\mathrm{MoS}_{2}$-stacked crystals were grown by APCVD in a two-zone furnace with a 2-inch diameter horizontal quartz tube. For growth, a $15 \mathrm{mg}$ mixture of molybdenum oxide $\left(\mathrm{MoO}_{3}\right)(\geqslant 99.5 \%$, Sigma-Aldrich, St Louis, MO, USA) and $\mathrm{NaCl}(\geqslant 99.99 \%$, Sigma-Aldrich) was loaded into a crucible located at the center of a high-temperature furnace. Sulfur ( $\mathrm{S})(0.8 \mathrm{~g}, \geqslant 99.5 \%$, Sigma-Aldrich, St Louis, MO, USA) powder was placed in the upstream region $15 \mathrm{~cm}$ away from the center of the $\mathrm{MoO}_{3}$ zone. Before CVD growth, a 300-nm-thick $\mathrm{SiO}_{2}$ / $\mathrm{Si}$ substrate was cleaned first in water followed by acetone and finally in isopropyl alcohol and then dried using $\mathrm{N}_{2}$. The $\mathrm{Si} / \mathrm{SiO}_{2}$ wafer was placed on the top of the boat, facing the polished side downwards. The temperatures of the $\mathrm{MoO}_{3}$ and $\mathrm{S}$ zones were kept at 850 and $200{ }^{\circ} \mathrm{C}$, respectively. $\mathrm{Ar} / \mathrm{H}_{2}$ gas $(50 / 7.5 \mathrm{sccm})$ was introduced as a carrier gas and to create a reducing atmosphere to promote the reaction. The $\mathrm{S}$ zone was preheated and moved when the $\mathrm{MoO}_{3}$ zone reached $250^{\circ} \mathrm{C}$. The furnace was heated with a ramp rate of $35^{\circ} \mathrm{C}$ per min to the growth temperature and held there for $30 \mathrm{~min}$. After the growth, the furnace was cooled naturally to room temperature.

\section{Raman and PL characterization}

The CVD-grown sample was characterized using PL and Raman spectroscopies (Horiba Jovin Yvon, LabRAM Aramis) atomic force microscopy (AFM) imaging (Park system, NX-10). The diameter of the illumination volume at the focus of the PL and Raman measurements was approximately $350 \mathrm{~nm}$. Scattered light was collected through the objective used for illumination and guided to a 50-cm-long monochromator (equipped with a cooled CCD) through free space. Diffraction gratings with 600 and 1800 grooves $\mathrm{mm}^{-1}$ were used to collect the PL and Raman spectra, respectively. The 532-nm lines of a solid-state laser were used at intensities of less than $300 \mu \mathrm{W}$ in a commercial confocal microscope system equipped with a 0.9 -NA objective lens. With this power level of laser illumination, no physical damage or oxidation was expected to occur in the $\mathrm{MoS}_{2}$ film.

\section{Computational details}

The simulations were performed using density functional theory with the projector-augmented-wave method, ${ }^{19,20}$ as implemented in the Vienna ab initio simulation package code. ${ }^{21}$ The Perdew-Burke-Ernzerhof ${ }^{22}$ generalizedgradient-approximation exchange-correlation functional was used, and an energy cutoff of $500 \mathrm{eV}$ was used for the plane-wave expansion. In this calculation, an integration over the Brillouin zone was carried out using a $24 \times 24 \times 1$ Monkhorst-Pack $k$-point mesh for all considered systems and a vacuum region accounting for more than $20 \AA$. All geometries were optimized using the conjugate-gradient method (force less than $0.0001 \mathrm{eV} \AA^{-1}$ ) with a van der Waals (vdW) correction (optB88-vdW functional) ${ }^{23,24}$ on the interlayer distances. To obtain a more accurate electronic band structure over the conventional generalized-gradient-approximation functional, here, we used the computationally expensive Heyd-Scuseria-Ernzerhof (HSE06) ${ }^{25}$ hybrid functional with SOC.

\section{Nonlinear optical characterization}

A dual-mode erbium-doped fiber laser (Insight Deepsee Dual, Spectra-Physics, Santa Clara, CA, USA) was combined with a confocal microscope (Olympus, IX 83, Tokyo, Japan) to create multimodal (SHG and FWM) nonlinear optical images of the CVD-grown $\mathrm{MoS}_{2}$ flakes. The dual-mode laser housing emits a fundamental ultrafast $(80-\mathrm{MHz})$ pulse train $(1040 \mathrm{~nm}, \mathrm{FWHM} \sim 220 \mathrm{fs})$, which synchronously pumps inside optical-parametric oscillators to generate a wavelength-variable (from 680 to $1300 \mathrm{~nm}$ ) pulse train (FWHM $120 \mathrm{fs}$ ). The beam intensity was adjusted using a spiral-type neutral-density filter (Thorlabs, NDC-100C-4M, Newton, MA, USA). The time gap between two pulses was synchronized using a commercial translational stage (Sigma-Koki, SGSP46-500, Tokyo, Japan). Two spatially overlapped beams were directed using a dichroic mirror (Thorlabs, DMSP1000R, Newton, MA, USA) to the galvanometric $x-y$ directional mirror controlled by a scanning system (Olympus, Fluoview 1000, Tokyo, Japan) to achieve real-time image acquisition. They were also monitored by a CCD camera (PIXIS 100B, Princeton Instruments, Trenton, NJ, USA) assisted by a monochromator (Acton SP2300, Princeton Instruments, Trenton, NJ, USA) for wide-wavelength spectroscopy $(500 \sim 700 \mathrm{~nm})$.

The details of the nonlinear optical microscopy setup have been reported previously ${ }^{26,27}$. Briefly, we employed a fiber-optically amplified femtosecond laser (Insight Deepsee Dual, Spectra-Physics, Santa Clara, CA, USA) as an illumination source for the SHG and FWM imaging after being assembled on an inverted type microscope (Olympus, IX 83, Tokyo, Japan). We used a $\sim 1 \mathrm{~mW} 800 \mathrm{~nm}$ beam $(\sim 120 \mathrm{fs})$ and a $1 \mathrm{~mW} 1040 \mathrm{~nm}$ beam $(\sim 220 \mathrm{fs})$ to generate the SHG at $520 \mathrm{~nm}$ and FWM at $650 \mathrm{~nm}$, respectively. The beams were focused with a 1.35 NA UPlanFLN objective lens, and the produced SHG/ FWM signals were collected by a photomultiplier tube (3523, Hamamatsu, Shizuoka, Japan) in reflection mode using two commercial filters (FF520/15, FF650/40, Semrock, Rochester, USA). The typical spatial resolution of the SHG microscope was $\sim 300 \mathrm{~nm}$. 
a

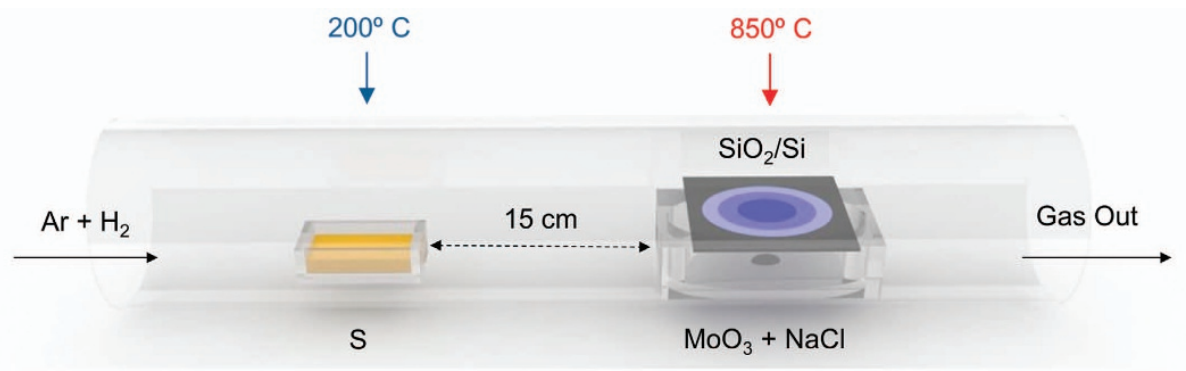

b
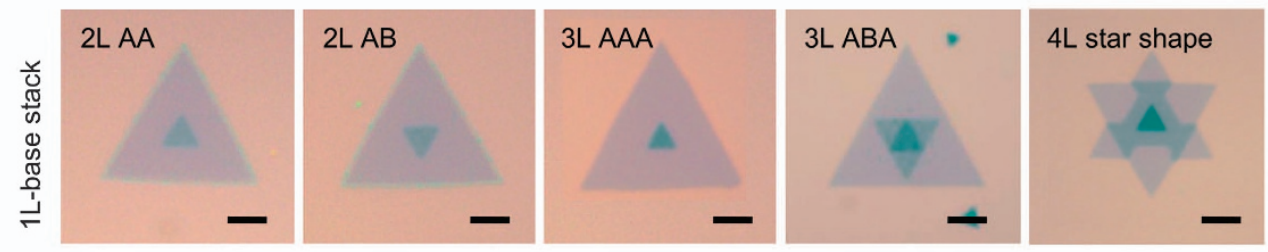

c
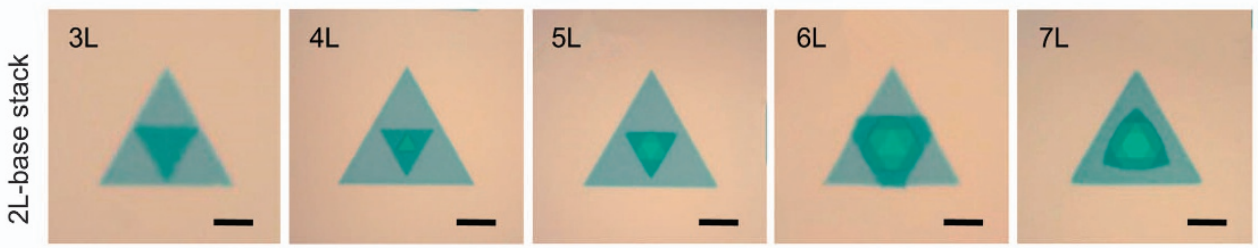

d
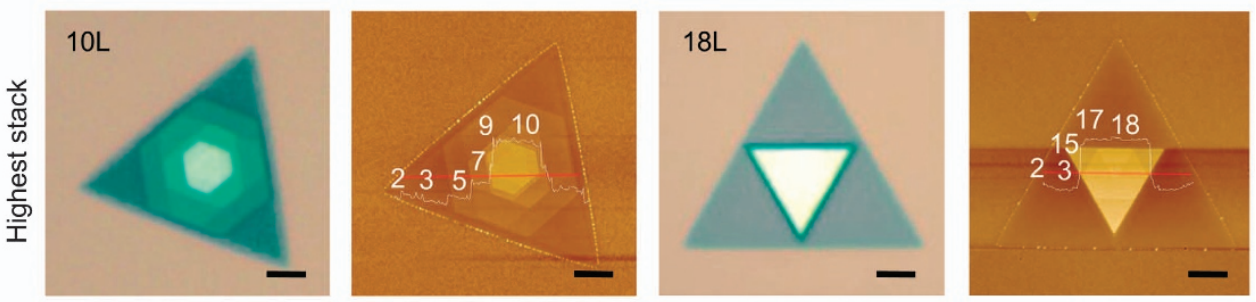

e
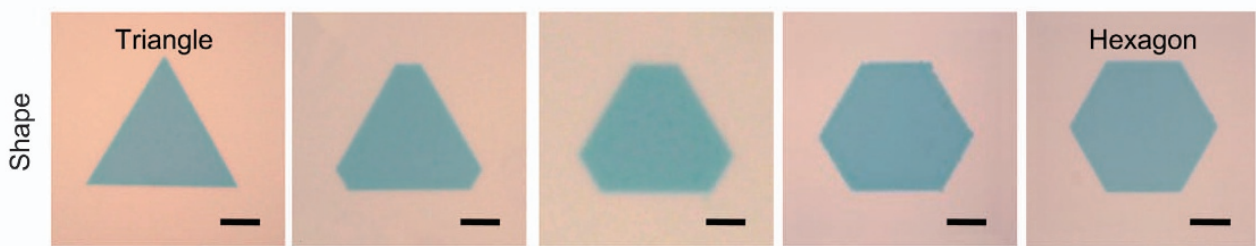

Figure 1 Stacking-oriented CVD fabrication of $\mathrm{MoS}_{2}$ multiple-layer systems. (a) Schematic representation of the synthesis of multi-stacked MoS 2 crystals via APCVD. (b) $2 \mathrm{~L}-4 \mathrm{~L}$ stacks of $\mathrm{MoS}_{2}$ crystals 'seeded' by monolayer systems. (c) $3 \mathrm{~L}-7 \mathrm{~L}$ stacks of $\mathrm{MoS}_{2}$ crystals seeded by bilayer systems. (d) Optical and AFM images for $10 \mathrm{~L}$ and $18 \mathrm{~L}$ stacked $\mathrm{MoS}_{2}$ crystals. All scale bars from (b) to (d) correspond to $5 \mu \mathrm{m}$. (e) Shape transformation of the MoS 2 crystals from triangles to hexagons. Scale bar of (e) corresponds to $4 \mu \mathrm{m}$. CVD, chemical vapor deposition; $\mathrm{MoS}_{2}$, molybdenum disulfide; APCVD, atmospheric-pressure chemical vapor deposition.

\section{RESULTS AND DISCUSSION}

\section{Synthesis of multi-stacked $\mathrm{MoS}_{2}$ crystals}

Figure 1a describes the synthesis strategy based on the layer-by-layer growth of multi-stacked $\mathrm{MoS}_{2}$ crystals by the APCVD technique. Briefly, the APCVD system consists of two zones for sulfur (S) and $\mathrm{MoO}_{3}+\mathrm{NaCl}$ powders, respectively. The boat contains a $15-\mathrm{mg}$ mixture of $\mathrm{MoO}_{3}$ and $\mathrm{NaCl}$ loaded in the center of a 2-inchdiameter horizontal quartz tube. The growth substrate (300-nm $\left.\mathrm{SiO}_{2} / \mathrm{Si}\right)$ is placed $\sim 10 \mathrm{~mm}$ above the precursor $\left(\mathrm{MoO}_{3}+\mathrm{NaCl}\right)$ with the polished face upside down (Figure 1a). Another boat containing $\mathrm{S}$ powder $(0.8 \mathrm{~g})$ is positioned in the upstream region $15 \mathrm{~cm}$ away from the precursor. The experimental section provides more details concerning the synthesis process. Note that the deposition range of the $\mathrm{MoS}_{2}$ crystals on the growth substrate is sectioned into four parts from the center to the edge (Supplementary Figure S1).
Generally, highly stacked crystal growth without using $\mathrm{NaCl}$ is possible only at temperatures $\geqslant 900^{\circ} \mathrm{C}$. With the aim of accelerating the reaction kinetics, we introduced $\mathrm{NaCl}$ as a growth promoter for the synthesis of multi-stacked $\mathrm{MoS}_{2}$ crystals. Mo-oxychlorides have a low sublimation temperature, owing to the fusion of $\mathrm{NaCl}$ and $\mathrm{MoO}_{3}$, followed by rapid vaporization, making it difficult to regulate the rate of the supply sources. This variable rate of evaporation leads to a very high precursor availability in the reaction zone, resulting in the formation of highly stacked $\mathrm{MoS}_{2}$ crystals with different stacking orientations. The uneven distribution of precursors from the center to the edge of the reaction zone helps in the formation of differently ordered highly stacked $\mathrm{MoS}_{2}$ crystals over the growth substrate (Supplementary Figure S1). Unlike the conventional $\mathrm{MoO}_{3}$ precursor with a high sublimation temperature of $\sim 700{ }^{\circ} \mathrm{C}$, herein, the primary growth precursors are $\mathrm{MoO}_{2} \mathrm{Cl}_{2}$ and $\mathrm{MoOCl}_{4}$ with sublimation temperatures of $\sim 100$ and $\sim 156^{\circ} \mathrm{C}$, respectively. The following 
equations give the possible reaction route:

$$
\begin{aligned}
& 5 \mathrm{MoO}_{3(\mathrm{~s})}+6 \mathrm{NaCl}_{(\mathrm{S})} \rightarrow 3 \mathrm{Na}_{2} \mathrm{MoO}_{4(\mathrm{~S})}+\mathrm{MoOCl}_{4(\mathrm{~g})} \\
& \quad+\mathrm{MoO}_{2} \mathrm{Cl}_{2(\mathrm{~g})} \\
& \mathrm{MoOCl}_{4(\mathrm{~g})}+\mathrm{MoO}_{2} \mathrm{Cl}_{2(\mathrm{~g})}+4 \mathrm{~S}_{(\mathrm{g})}+6 \mathrm{H}_{2(\mathrm{~g})} \rightarrow 2 \mathrm{MoS}_{2(\mathrm{~s})} \\
& \quad+6 \mathrm{HCl}_{(\mathrm{g})}+3 \mathrm{H}_{2} \mathrm{O}_{(\mathrm{g})}
\end{aligned}
$$

Solid $\mathrm{MoO}_{3}$ fuses with $\mathrm{NaCl}$ at $600{ }^{\circ} \mathrm{C}$ to form $\mathrm{Na}_{2} \mathrm{MoO}_{4}, \mathrm{MoO}_{2} \mathrm{Cl}_{2}$ and $\mathrm{MoOCl}_{4}{ }^{28} \mathrm{Na}_{2} \mathrm{MoO}_{4}$ is a solid and completely non-volatile product, whereas $\mathrm{MoO}_{2} \mathrm{Cl}_{2}$ and $\mathrm{MoOCl}_{4}$ are both volatile. These Nacontaining intermediates, which are similar to PTAS (perylene3,4,9,10-tetracarboxylic acid tetrapotassium salt) and PTCDA (perylene-3,4,9,10-tetracarboxylic dianhydride) seeds, provide nucleation sites on the substrate and then are sulfurized into the oxi-chalcogenide particles. In other words, these Na-intermediate compounds help reduce the energy of the reaction (energy required for the reactants to form the final product) required to form the final product $\left(\mathrm{MoS}_{2}\right)$ by lowering the overall growth temperature. In the following step, the particles are converted into highly stacked $\mathrm{MoS}_{2}$ crystals with different stacking orientations by exposure to continuous sulfur vapor. At the same time, the addition of $\mathrm{Na}$ atoms enlarges the space between the sheets and enhances the separation process, resulting in a solid-vapor reaction. The amount of $\mathrm{NaCl}$ has a variable influence on nucleation and growth under given conditions. With an increase of the $\mathrm{NaCl}$ amount, multi-stacked crystal growth dominates instead of monolayer $\mathrm{MoS}_{2}$ domain growth, which is an indication of the rapid growth rate. Furthermore, the effect of $\mathrm{NaCl}$ becomes more prominent as the temperature increases, because the rate of the chemical reaction is directly proportional to the temperature. Compared to the reaction temperature used for the synthesis of highly stacked $\mathrm{MoS}_{2}$ crystals using the conventional method $\left(\geqslant 950^{\circ} \mathrm{C}\right)$, the temperature applied in this work is dramatically reduced by the addition of the $\mathrm{NaCl}$ growth promoter $\left(850^{\circ} \mathrm{C}\right)$. In conclusion, by controlling the amounts of Mo precursor and $\mathrm{NaCl}$ at a particular temperature, the stacking order can be controlled. An XPS study was carried out for these stacked crystals, and it revealed no characteristic peaks for Nals and $\mathrm{Cl} 2 \mathrm{p}$, suggesting that the stacked $\mathrm{MoS}_{2}$ crystals are free from contamination by alkali metals and halogen atoms ${ }^{17}$ (Supplementary Figures S4c and d). In addition, energy-dispersive spectroscopy analysis also shows the absence of any trace $\mathrm{Na}$ and $\mathrm{Cl}$ in the as-grown $\mathrm{MoS}_{2}$ crystals (Supplementary Table S1). The Mo metal in $\mathrm{MoS}_{2}$ has no available d-electrons to fill the $\mathrm{d}_{\mathrm{z}}{ }^{2}$ level, resulting in an exclusive octahedral Mo geometry to offer a more stable structure. The $\mathrm{AB}(\mathrm{A} . .$.$) -stacking$ sequence has hexagonal close packing with a coordination number of 12 and packing efficiency of $74 \%$, whereas $\mathrm{AA}(\mathrm{A} . .$.$) stacking results in$ simple cubic packing with a coordination number of 6 and packing efficiency of $52 \%$. Higher coordination numbers and packing efficiencies make $\mathrm{AB}(\mathrm{A} \ldots)$ stacking energetically more stable and preferred over $\mathrm{AA}(\mathrm{A} \ldots)$. Schematic representations of the $2 \mathrm{H}, 3 \mathrm{R}$ and mixed-type stacking orders for $2 \mathrm{~L}$ and $3 \mathrm{~L}$ are shown in Supplementary Figure S3.

The $\mathrm{AB}(\mathrm{A} \ldots)$-stacked $\mathrm{MoS}_{2}$ crystals with the lowest formation energy are dominant among those grown by APCVD, indicating that our growth conditions favor this stacking over other high-energy stacking sequences (false stacking) because of the higher-energy barriers required by those configurations. ${ }^{11}$ Figure $1 \mathrm{~b}$ shows the $\mathrm{MoS}_{2}$-monolayer seeded stacking crystals only up to $4 \mathrm{~L}$, as the precursor concentration gradient is the lowest in this area. Interestingly, in some isolated part of the substrate, star-shaped stacking was also observed, as shown in Supplementary Figure S2a. Upon moving toward the center, the precursor concentration gradient increases the chance of forming isolated $\mathrm{MoS}_{2}$-bilayer seeded multi-stacked crystals (multi-stacked crystals grown on an individual bilayer $\mathrm{MoS}_{2}$ unit) with stacking orders up to 7L (Figure 1c). Furthermore, when the precursor concentration gradient peaks near the summit point, multi-stacked $\mathrm{MoS}_{2}$ crystals with the highest stacking order are observed. Figure 1d illustrates the OM and AFM images of $10 \mathrm{~L}$ and $18 \mathrm{~L}$ multi-stacked $\mathrm{MoS}_{2}$ crystals with different orientations, together with the corresponding AFM height profiles.

In addition to the triangular shape, other shapes were also formed during the growth of the multi-stacked $\mathrm{MoS}_{2}$ crystals. By varying the Mo:S ratio, the shapes of the $\mathrm{MoS}_{2}$ crystals evolve from a triangle to a truncated triangle and finally become hexagonal ${ }^{29,30}$, as shown in Figure 1e. The shape transformation process along the direction perpendicular to the carrier-gas flow stipulates that the Mo:S ratio change is the primary reason for the shape transformation. All conditions, including the growth temperature and S concentration, are constant in this direction, except for the different masses of Mo. Thus, the Mo:S ratio plays an important role and could be a determining parameter.

The confined Mo:S atomic ratio controls the final shape of the $\mathrm{MoS}_{2}$ crystals, as explained by the Wulff construction principle, ${ }^{29,30}$ which states that specific crystal shapes are preferred over others depending on their surface energies. In other words, the surface energy and its dependence on the crystallographic orientation can define the equilibrium shape of a crystal. When the Mo:S atomic ratio matches the stoichiometric ratio of $\mathrm{MoS}_{2}$ (1:2) (here 0.51), Mo and S terminations follow the same growth rates. Thus, the dominant shape of the $\mathrm{MoS}_{2}$ crystals is hexagonal (Growth I). However, with Mo: $\mathrm{S} \geqslant 1: 2$, a Mo-rich atmosphere results. Once the S-terminated edges grow rapidly and finally disappear, the entire crystal will possess Moterminated edges with a triangular shape (Growth II) (here; 0.45). However, when the Mo:S atomic ratio is between growths I and II (here; 0.48), the $\mathrm{MoS}_{2}$ crystal will transform into a truncated triangle. ${ }^{28,31}$ Additional evidence supporting the above information is given by energy-dispersive spectroscopy analysis (Supplementary Table). Based on the APCVD system, multi-stacked $\mathrm{MoS}_{2}$ crystals can be formed not only in the shape of triangles but also in the shapes of truncated triangles and hexagons, as shown in Supplementary Figures $2 \mathrm{~b}$ and $\mathrm{c}$. We further describe the transformation of the $\mathrm{MoS}_{2}$ crystals from a monolayer to complete bilayer in Supplementary Figure S2d. This bilayer forms when the top layer entirely extends to the edges of the bottom layer. A low Mo-concentration gradient at the monolayer site causes a low stacking order (3-4L), whereas a very high Moconcentration gradient near the center results in bilayer seeded highly stacked $\mathrm{MoS}_{2}$ crystals ( $\geqslant 18 \mathrm{~L}$ ).

In the APCVD system, a $\mathrm{MoS}_{2}$-stacking order change is possible along the $X$ - (carrier-gas flow) and $Y$-axes (perpendicular to the gas flow) because of the change in the Mo-concentration gradient along both the axes. The precursor concentration gradient decreases toward the edge of the deposited substrate as the deposition site becomes farther from the precursor, resulting in a reduced stacking order. The thickness of the obtained crystals can be tuned from $1 \mathrm{~L}$ to $\geqslant 18 \mathrm{~L}$ by controlling the amount of precursor and growth temperature. Moreover, the temperature for introducing sulfur is also a critical parameter for the well-defined layer-by-layer stacking of $\mathrm{MoS}_{2}$ with an ideal size and shape. The introduction of sulfur into the Mo precursor zone is a determining factor for the size of the $\mathrm{MoS}_{2}$ crystals. By controlling it, $\mathrm{MoS}_{2}$ crystals with sizes $>100 \mu \mathrm{m}$ can also be fabricated using APCVD growth. Here, the introduction of sulfur before the precursor zone reaches $150^{\circ} \mathrm{C}$ results in the formation of bulk $\mathrm{MoS}_{2}$ that does 
not sublime under the given conditions but leads to no crystal growth on the substrate. In contrast, early introduction of sulfur leads to smaller crystals, and delayed introduction forms large-area bilayer seeded multi-stacked $\mathrm{MoS}_{2}$ crystals. In conclusion, this growth method of stacked $\mathrm{MoS}_{2}$ crystals with controlled orientation is scalable to large-size crystals. The growth temperature also governs the stacking order and pattern. Below $850{ }^{\circ} \mathrm{C}$, the stacking order decreases with a high probability of monolayer-based stacked crystals covering isolated monolayer crystals in some areas. When the growth temperature increases above $850^{\circ} \mathrm{C}$, the stacking order increases, forming bilayer seeded crystals with some layers reaching up to $\sim 15-20 \mathrm{~L}$ with the formation of completely grown $3 \mathrm{~L}$ or $4 \mathrm{~L} \mathrm{AA}(\mathrm{A} \ldots)$ - and $\mathrm{AB}(\mathrm{A} \ldots)$ stacked $\mathrm{MoS}_{2}$ crystals. ${ }^{30}$ Furthermore, this growth is governed by a terrace-growth mechanism, wherein the triangles stack up layer by layer and shrink gradually to the center. During this growth process, the upper layers are formed later and have a shorter time to grow. Therefore, the lateral layer size decreases with an increasing height, and monolayer-by-monolayer-stacked $\mathrm{MoS}_{2}$ crystals are finally formed. ${ }^{29-34}$

Stacking-oriented phonon frequencies in the Raman spectra

We critically examined the high-energy phonons in the Raman spectra of multi-stacked $\mathrm{MoS}_{2}$ crystals and exploited the stacking-induced structural changes and interlayer vdW interactions. As illustrated in
Figure 2a, a $\mathrm{MoS}_{2}$ crystal consists of two well-defined, strong Raman bands attributed to lattice vibrations in two specific directions, which are denoted as in-plane $\left(\mathrm{E}_{2 \mathrm{~g}}{ }^{1}\right)$ and out-of-plane $\left(\mathrm{A}_{1 \mathrm{~g}}\right)$ vibrations. ${ }^{1,2,7-13,35-39}$ Since the peak positions and frequency differences between $\mathrm{E}_{2 \mathrm{~g}}{ }^{1}$ and $\mathrm{A}_{1 \mathrm{~g}}$ are known to reflect the spectroscopic figures induced by the shape and thickness variations of multi-stacked $\mathrm{MoS}_{2}$ crystals, we first compared the Raman spectra of the monolayer and bilayer hexagonal (dotted line) and triangular (solid line) crystals, as shown in Figure 2a. Both the hexagons and triangles exhibited welldefined $\mathrm{A}_{1 \mathrm{~g}}$ and $\mathrm{E}_{2 \mathrm{~g}}{ }^{1}$ Raman modes. The peak positions of the $\mathrm{A}_{1 \mathrm{~g}}$ modes of both the monolayer crystals coincide, whereas the corresponding $\mathrm{E}_{2 \mathrm{~g}} 1$ mode of the hexagons was noticeably softened compared to that of the triangles. However, in the hexagonal bilayers, both the $\mathrm{E}_{2 \mathrm{~g}}{ }^{1}$ and $\mathrm{A}_{1 \mathrm{~g}}$ modes softened by approximately $1.5 \mathrm{~cm}^{-1}$ compared to that of the triangular bilayers. Dislocation cores, vacancies or lattice mismatches are known to be highly capable of forming during crystal growth, resulting in subtle differences in the phonon frequencies of the Raman spectra. A recently reported result suggests that the presence of either an $\mathrm{S}$ or $\mathrm{W}$ vacancy is more likely to lower the Raman frequency in a hexagonal $\mathrm{WS}_{2}$ single crystal. ${ }^{36}$ In this manner, the relatively softened $\mathrm{E}_{2 \mathrm{~g}}{ }^{1}$ vibrations in both hexagonal mono- and bilayer $\mathrm{MoS}_{2}$ likely result from an effect of vacancy induced defect states.
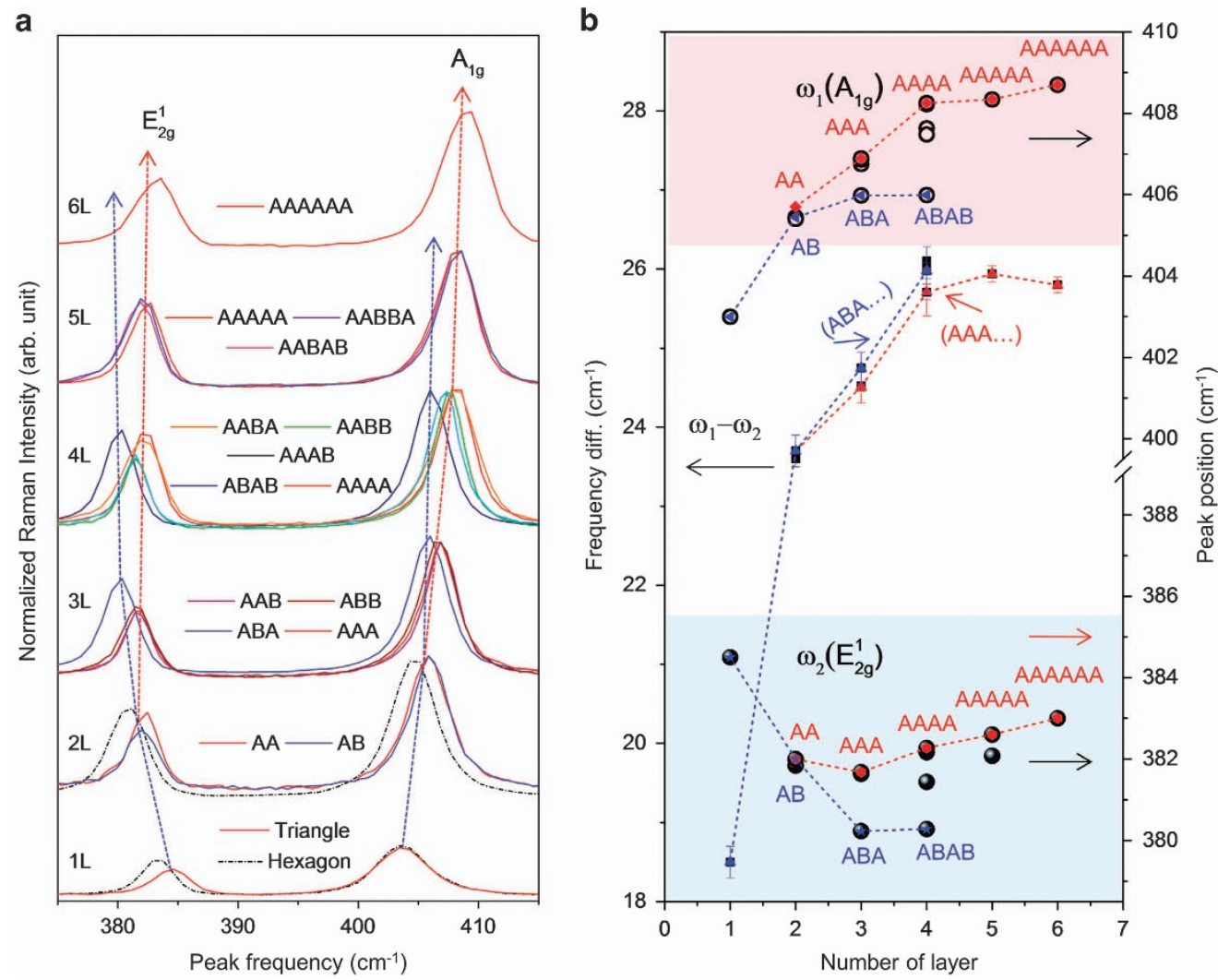

Figure 2 Shape- and stacking-orientation-dependent Raman spectroscopic characterization of the MoS 2 crystals as a function of the layer thickness. (a) Raman spectra for various layer thicknesses and stacking orientations achieved using a 0.1-mW, 532-nm laser excitation source. All spectra were taken from a triangular-shaped sheet (solid lines), except for two hexagonal cases (dashed lines). Guiding arrows are inserted to show the trends of the peak changes among similar stacking orientation species. Both the $E_{2 g}{ }^{1}$ and $A_{1 g}$ modes of the $A A(A$...)-type stacking configurations (red dashed line) are blueshifted compared to the $\mathrm{AB}(\mathrm{A}$...)-type stacking configurations (blue dashed line) for layer thicknesses greater than $2 \mathrm{~L}$. (b) Peak frequencies (right axis) of the $E_{2 g}{ }^{1}$ and $A_{1 g}$ phonon vibrations and their peak-to-peak differences (left axis) are plotted as functions of the layer thickness. Note that both the $E_{2 g}{ }^{1}$ and $A_{1 g}$ modes of the 'fault-ordered' $A A B(A . .$.$) -type stacking configurations possess Raman frequencies (black balls) located between the A A(A .$.$) - and A B(A . .$.$) -$ type stacking configurations. 
a
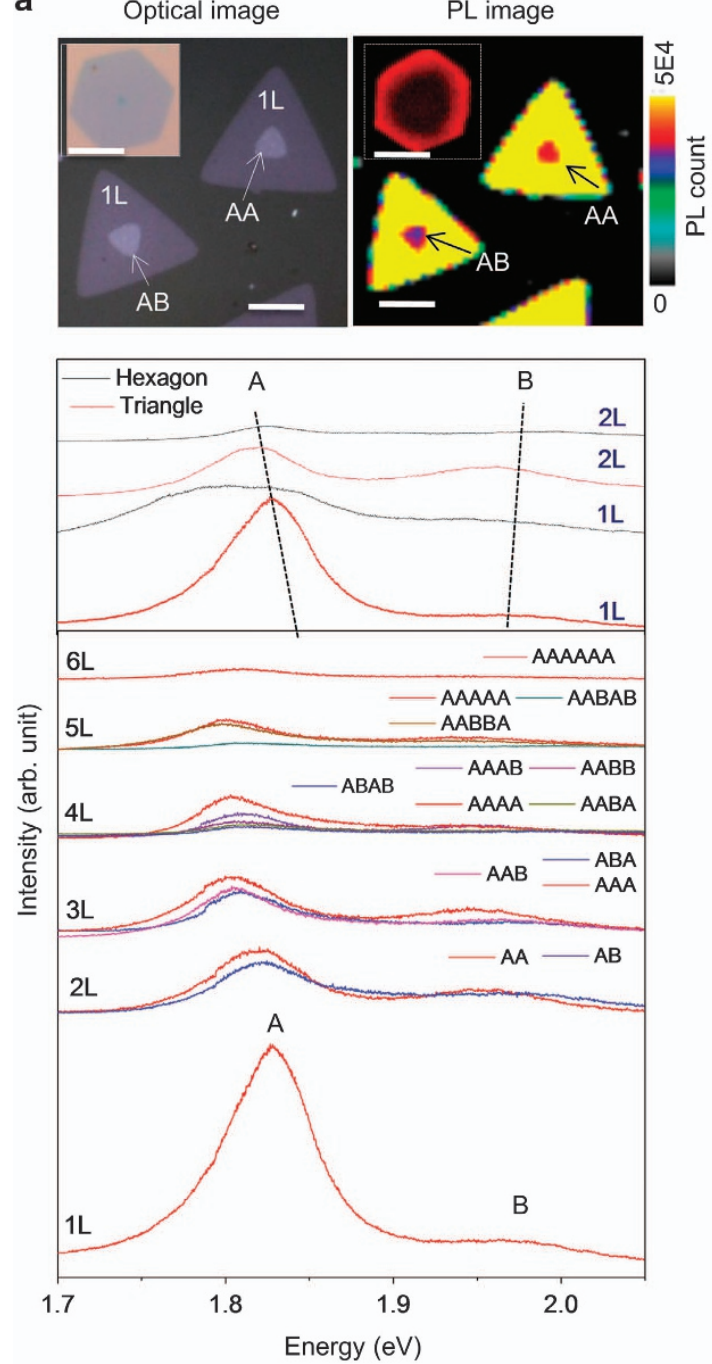

b

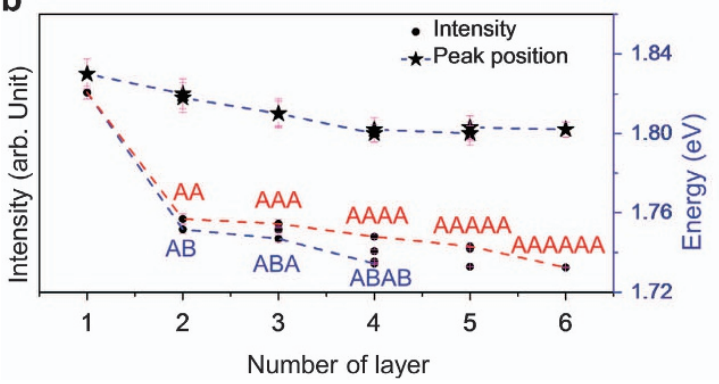

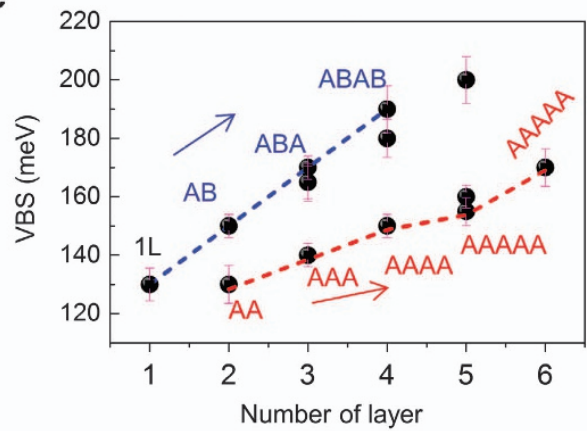

d

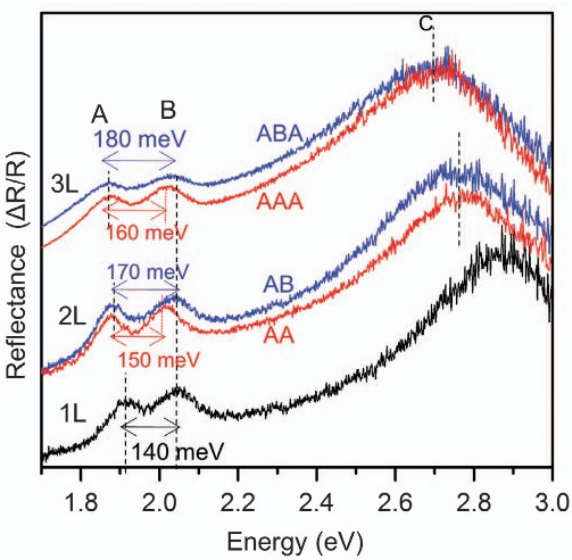

e

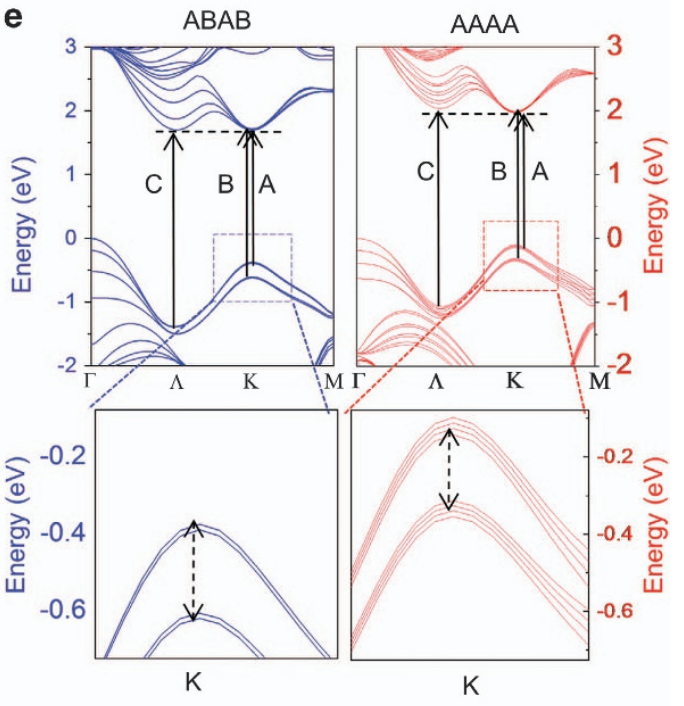

Figure 3 Effects of the shape and stacking orientation on the electronic band structures of multilayer $\mathrm{MoS}_{2}$ crystals. (a) Optical and photoluminescence (PL) mapping images of the flakes containing $1 \mathrm{~L}, 2 \mathrm{~L}-\mathrm{AA}$ and $2 \mathrm{~L}-\mathrm{AB}$ triangular- and hexagonal-shaped crystals are shown at the top (scale bar is $3 \mu \mathrm{m}$ ). All spectra and mappings are acquired using 532-nm-laser illumination. The PL intensity of the triangular pit was higher than those of the hexagonal crystals. The shape- and stacking-orientation-dependent PL spectra are shown at the bottom panel as a function of the layer thickness. The positional variations of the $\mathrm{A}$ and $\mathrm{B}$ peaks are guided by black dashed lines for the hexagons and triangles, which were roughly described with an increasing thickness from $1 \mathrm{~L}$ to 6L. (b) The positional variations in the A peak (right) and integrated PL intensities (left) were dependent on the layer thickness and stacking orientation configuration. The monolayer triangular crystal demonstrated the highest PL intensity due to its direct band gap. The red and blue dashed lines guide the intensity variations of the AAA... and ABA... stacking cases, respectively. (c) Valence-band splitting (VBS) of the variously stacked crystals plotted as a function of the number of layers. (d) Absorption spectra obtained from the 1L, 2L-AA, 2L-AB, 3L-ABA and 3L-AAA flakes with the A, B and C excitons clearly resolved. (e) Calculated band structures of the ABAB- (left panel) and AAAA-stacked (right panel) 4L-MoS 2 systems. The VBS near the K point is magnified to better show the direct-band-gap transitions. The estimated error bar is provided in the corresponding graph with a pink color. 
We also studied the Raman spectral changes of triangular $\mathrm{MoS}_{2}$ crystals with regards to various stacking orientations and thicknesses ranging from $1 \mathrm{~L}$ to $6 \mathrm{~L}$. Raman spectra with distinct shifts in the $A_{1 g}$ and $\mathrm{E}_{2 \mathrm{~g}} 1$ modes are demonstrated in Figure 2a. The layer-thickness- $/$ orientation-dependent variations of the $\mathrm{E}_{2 \mathrm{~g}}{ }^{1}$ and $\mathrm{A}_{1 \mathrm{~g}}$ vibrational modes are summarized in Figure $2 \mathrm{~b}$. Blue and red dashed lines indicated the trends of the Raman frequencies for the $\mathrm{AB}(\mathrm{A} \ldots)$ - and $\mathrm{AA}(\mathrm{A} . .$.$) -stacking configurations, respectively. Here, the Raman peak$ positions of the mixed orientations are located in between the AA (A...)- and $\mathrm{AB}(\mathrm{A} . .$.$) -stacking configurations, as indicated by the$ isolated balls $\left(\mathrm{E}_{2 \mathrm{~g}}{ }^{1}\right)$ or circles $\left(\mathrm{A}_{1 \mathrm{~g}}\right)$ in Figure $2 \mathrm{~b}$.

The thickness-dependent shifting of the Raman spectra for the $\mathrm{AB}$ (A...) vdW-layer system manifests the similarity of the developed interlayer coupling behavior of the exfoliated natural crystals. ${ }^{1,2,35}$ As we observed, the $\mathrm{A}_{1 \mathrm{~g}}$ mode blueshifted (stiffened), the $\mathrm{E}_{2 \mathrm{~g}}{ }^{1}$ mode redshifted (softened), and the separations between the $\mathrm{E}_{2 \mathrm{~g}}{ }^{1}$ and $\mathrm{A}_{1 \mathrm{~g}}$ modes gradually increased with an increasing layer thickness. The blueshift of the $A_{1 g}$ mode with an increasing layer number indicates stronger interlayer vdW interactions, whereas the redshift of the $E_{2 \mathrm{~g}}{ }^{1}$ mode arises from dielectric-screening-related long-range Coulomb interactions. ${ }^{1,2,35}$ In contrast, both the $\mathrm{A}_{1 \mathrm{~g}}$ and $\mathrm{E}_{2 \mathrm{~g}}{ }^{1}$ Raman modes of the $\mathrm{AA}(\mathrm{A} . .$.$) -stacked samples with more than two layers are stiffened$ compared to those of the $\mathrm{AB}(\mathrm{A} . .$.$) -stacked crystals. We note that the$ peak frequency differences between the two modes for $\mathrm{AA}(\mathrm{A} . .$.$) -type$ and $\mathrm{AB}(\mathrm{A} . .$.$) -type stacking are similar, suggesting nearly equal shifting$ of the $E_{2 g}^{1}$ and $A_{1 g}$ frequency modes, as marked as red and blue dashed lines, respectively (Figure $2 \mathrm{~b}$ ). The shifting tendency of the phonon frequencies depending on the stacking orientations is consistent with the previously calculated result at the gamma point. ${ }^{11}$ More details concerning the stacking-order dependence of the Raman peak positions for each $\mathrm{MoS}_{2}$ crystal $(2 \mathrm{~L}-5 \mathrm{~L}$ ) can be found in Supplementary Figure S5. This interesting result reveals that a reduction in the interlayer distances occurs in the $\mathrm{AA}(\mathrm{A} \ldots)$-stacked crystals compared to the $\mathrm{AB}(\mathrm{A} . .$.$) -stacked crystals, which is similar to$ a previously reported result. ${ }^{11}$ An experimental study carried out by Yan et al. ${ }^{11}$ demonstrated that the $\mathrm{E}_{2 \mathrm{~g}}{ }^{1}$ mode for the AAA-trilayer was barely blueshifted, while the frequency of the $A_{1 g}$ mode was stiffened with respect to the $\mathrm{ABA}$-trilayer. The $\mathrm{E}_{2 \mathrm{~g}}{ }^{1}$ mode preservation against layer stacking in the AAA-type crystal, as reported in Yan et al. ${ }^{11}$ might be related to the structural diversity induced by the fabrication method, as Raman spectroscopy of the CVD crystal is highly sensitive to the growth conditions and quality of the sample. We believe that our observation of considerable phonon stiffening for both the $\mathrm{E}_{2 \mathrm{~g}}{ }^{1}$ and $\mathrm{A}_{1 \mathrm{~g}}$ modes in the $\mathrm{AA}(\mathrm{A} . .$.$) -stacked crystals becomes visible due to$ the negligible contributions of CVD-induced structural defects.

\section{Stacking-oriented excitonic behavior in the PL spectra}

To understand the excitonic behavior of the various stacking-oriented layers with different geometries, we compared the PL emission spectra of the hexagonal and triangular $\mathrm{MoS}_{2}$ crystals, as shown in Figure 3a. The PL mapping images show that the hexagonal crystals has nonuniform and edge-prevailing PL, while the triangular crystal has uniform PL from the center-to-edge regions. A lower PL intensity in the inner region of the hexagonal sample is mainly ascribed to the influence of defect states localized in the inner region. As illustrated by a recent study regarding a hexagonal $\mathrm{WS}_{2}$ monolayer, defect states can be a key factor in reducing the PL emission in a localized fashion. ${ }^{36}$ Moreover, the tailored PL intensity is also related to the band structure change via an extra-strain effect induced by merged defects. ${ }^{37}$ The broadened and redshifted PL spectrum of the hexagonal monolayer compared to that of the triangular monolayer indicates the possible involvement of defect-assisted quenching in the PL emission. ${ }^{36}$ In fact, the activity of those defect states can be diminished using a nonoxidizing organic superacid, such as bis(trifluoromethane)sulfonimide, providing a drastic enhancement of the PL emission. ${ }^{40,41}$ Thus, to reaffirm that the decreased PL intensity in our hexagonal crystal originates from defects, we dipped the samples in a bis(trifluoromethane)sulfonimide solution for $1 \mathrm{~h}$ and found that the PL emissions drastically recovered in both the triangles and hexagons. The degree of enhancement was more severe in the hexagon than the triangle, implying that more defect states initially existed in the hexagonal $\mathrm{MoS}_{2}$ crystals. The corresponding results are provided in Supplementary Figure S6.

We further characterized the PL emission spectra from $1 \mathrm{~L}$ to $6 \mathrm{~L}$ for various stacking configurations (Figure $3 \mathrm{a}$, bottom panel). The monolayer exhibited the strongest PL intensity as a result of the vertical quantum-confinement effect induced by the indirect-to-direct band-gap crossover in a single-layer limit. ${ }^{1-3}$ For the multilayer systems, each thick crystal also exhibited stacking-orientationdependent PL behaviors. The maximum PL intensity was observed for the $\mathrm{AA}(\mathrm{A} . .$.$) -type stacking, followed by the mixed-type stacking,$ and the minimum $\mathrm{PL}$ was observed in the $\mathrm{AB}(\mathrm{A} \ldots)$-type stacked crystals. Simultaneously taking PL images of $2 \mathrm{~L}-\mathrm{AA}$ and $2 \mathrm{~L}-\mathrm{AB}$ shown in the top panel of Figure 3a clearly exhibited the improved PL emission from the AA-stacked crystal. The variations of the peak positions and integrated PL intensities as a function of the layer number are displayed in Figure $3 \mathrm{~b}$. The related PL intensities gradually decreased from $1 \mathrm{~L}$ to $6 \mathrm{~L}$. The excitonic position, that is, the direct optical band gap, was unchanged among the stacking orientations but monotonically decreased from $1 \mathrm{~L}$ to $3 \mathrm{~L}$ in a manner similar to those observed in previous studies. ${ }^{1,2,10-13}$ Interestingly, for the AA(A...)stacking configuration for greater than $4 \mathrm{~L}$, the optical band gap was slightly increased (by $\sim 5-10 \mathrm{meV}$ ) compared to those of the other stacking orders. Detailed comparisons between the band gaps and integrated PL intensities from the $2 \mathrm{~L}$ to $5 \mathrm{~L}$ crystals are summarized with regards to the different stacking orders in Supplementary Figure S7. Furthermore, we studied the PL and peak positional change of the indirect bands in the $\mathrm{AA}(3 \mathrm{R})$ - and $\mathrm{AB}(2 \mathrm{H})$-stacked bilayer crystals. The indirect band gaps in both cases were very similar, except for being slightly broadened for the AA bilayer (Supplementary Figure S8). The observed PL spectra for the indirect band gaps of the CVD-grown $\mathrm{AA}$ and $\mathrm{AB}$ bilayers indicate the interlayer electronic coupling strength, while the slightly broadened bandwidth of AA suggests stronger interlayer coupling. ${ }^{12,42}$

Next, the valence-band splitting (VBS) of the $\mathrm{MoS}_{2}$ crystals was studied as a function of the layer-number thickness and stacking-order configuration. Essentially, the amount of VBS was measured as the distance between the A- and B-peak energies observed in the PL or absorption spectra. Figure $3 \mathrm{c}$ shows the VBS tendencies from the $1 \mathrm{~L}$ to $6 \mathrm{~L}$ crystals for different orientations measured from the PL spectra. The gradual increase in the VBS with an increasing layer thickness is in good agreement with previous results. ${ }^{1,2}$ First, for $1 \mathrm{~L} \mathrm{MoS}_{2}$, SOC contributes to the VBS because the 1L crystal lattice has broken inversion symmetry. On the other hand, there is an even-odd variation in the structural symmetry of the $\mathrm{AB}(\mathrm{A} . .$.$) -stacked multi-$ layer: broken inversion symmetry is present (absent) in films with an odd (even) number of layers. Therefore, the major contribution to VBS is SOC for an odd number of layers, while for an even number of layer, the dominant effect is interlayer interactions. ${ }^{1-3,16,17}$ However, the structural symmetry in the $\mathrm{AA}(\mathrm{A} \ldots)$-stacked multilayers is independent of the even-odd oscillation with the number of layers: all the layers should have broken inversion symmetry, ${ }^{10-13}$ and thus, 
the observed VBS is mainly contributed by SOC. As shown in Figure $3 c$, the VBS phenomena exhibited a stacking-orientation dependence, with the $\mathrm{AB}(\mathrm{A} . .$.$) -stacked crystal showing larger VBS$ than the $\mathrm{AA}(\mathrm{A} . .$.$) -stacked crystal. This unique result under \mathrm{AA}(\mathrm{A} . .$.$) -$ stacking is observed as a result of the stacking-order-dependent shifting of the A and B excitonic PL peak positions. The A-peak position did not vary much until $4 \mathrm{~L}$ and then slightly increased for AA (A...)-stacking. Meanwhile, the B peak gradually redshifted for AA (A...)-stacking, leading to reduced VBS as the layer thickness increased. The stacking-orientation-dependent A- and B-peak positions and their differences for $2 \mathrm{~L}-5 \mathrm{~L}$ are provided in Supplementary Figure S7. Further discussion on the nature of the splitting will be provided later in combination with the density functional theory calculation results.

A similar VBS trend was also observed in the differential-reflectance spectra (Figure 3d). We performed differential-reflectance measurements for the $1 \mathrm{~L}, 2 \mathrm{~L}-\mathrm{AA}, 2 \mathrm{~L}-\mathrm{AB}, 3 \mathrm{~L}-\mathrm{ABA}$ and $3 \mathrm{~L}-\mathrm{AAA}$ stacking crystals after loading these crystals onto a transparent glass substrate. Reflection spectra of thin films measured from a transparent medium can be well interpreted as absorption spectra. ${ }^{1,2}$ The energy gaps observed in the PL spectra are highly sensitive to small changes associated with the doping level or defect states in the absorption bands and can capture the sources of inaccuracies in band-gap estimations. ${ }^{1,2,41}$ In addition, absorption spectra allow the evolution of the electronic band structures in a broad energy range, including high-energy excitons. As illustrated, the absorption bands for the $1 \mathrm{~L}$, 2L-AA, 2L-AB, 3L-ABA and 3L-AAA stacking crystals clearly show A, $\mathrm{B}$ and $\mathrm{C}$ excitons (Figure $3 \mathrm{~d}$ ). The $\mathrm{A}$ and $\mathrm{B}$ peaks are lower-energy excitons created by the splitting of the topmost valence bands. On the other hand, the $\mathrm{C}$ peak located at the high-energy state is induced by the electronic transition from band nesting. ${ }^{8}$ The overall features of the absorption spectra taken from the $1 \mathrm{~L}, \mathrm{AB}$ bilayer and ABA-trilayer samples replicate the results observed in the literature. ${ }^{1,2,8}$ However, for the 2L-AA and 3L-AAA stacking orders, different VBS natures and peak positions of the related excitons emerge. The relatively smaller VBS of the $\mathrm{AA}(\mathrm{A} \ldots)$-stacking than that of the $\mathrm{AB}(\mathrm{A} \ldots)$-stacking observed in the PL study is also consistently observed in the absorption study. Notably, the optical band gap represented by the A-peak position remains unchanged for both the $\mathrm{AA}(\mathrm{A} \ldots)$ and $\mathrm{AB}$ (A...)-stackings, clearly complying with the weak direct band-gap changes originating from the stacking orientations, as suggested by the PL studies. ${ }^{3,11,12,42}$ In contrast, the B-peak positions of the AA and AAA stackings have softened by approximately $20 \mathrm{meV}$, and thus, the VBS values in the $\mathrm{AA}(\mathrm{A} \ldots)$-stacked bi- and trilayers are reduced compared to those in the $\mathrm{AB}(\mathrm{A} . .$.$) bi- and trilayers. In addition, as$ shown in Figure $3 \mathrm{~d}$, the $\mathrm{C}$ peak barely changes with the stacking orientation. The gap of the $\mathrm{AA}(\mathrm{A} . .$.$) -stacked bi- or trilayers is$ approximately $5 \sim 10 \mathrm{meV}$ wider than that of the corresponding $\mathrm{AB}$ (A...)-stacked bi- or trilayers. We believe that this tendency of VBS and electronic band structure will be maintained in thicker AA(A...)and $\mathrm{AB}(\mathrm{A} . .$.$) -stacked crystals as well.$

\section{Stacking-oriented excitonic effects and valence-band splitting revealed by theoretical calculations}

To understand the dependence of the electronic structure on the stacking orientation, we performed density functional theory calculations of the $3 \mathrm{~L}$ and $4 \mathrm{~L}$ crystals with $\mathrm{AA}(\mathrm{A} . .$.$) - and \mathrm{AB}(\mathrm{A} \ldots)$-stacking (Figure 3e). We used both the HSE06 and Perdew-Burke-Ernzerhof functionals including SOC and investigated the electronic band structures. Details of the theoretical results are provided in the Supplementary Information (Supplementary Figures S9-S11). As indicated in Figure 3e, the valence- and conduction-band edges of the 4L-AAAA-stacking crystal are relatively higher than those in the $4 \mathrm{~L}-\mathrm{ABAB}$ crystal and shifted by the same amount $(\sim 250 \mathrm{meV})$. Hence, any change in the net direct band gap value is compensated. Consequently, the band gaps remained constant in both cases. This up-shifting of the corresponding valence and conduction bands is also applicable for the 3L-AAA crystal, as shown in Supplementary Information (Supplementary Figures S9 and S11). Therefore, we concluded that the direct band gap is independent of the stacking orientation. However, the indirect band gap seems to change slightly between the $\mathrm{AA}(\mathrm{A} \ldots)$ - and $\mathrm{AB}(\mathrm{A} \ldots)$-stacking orientations. For $\mathrm{AB}$ (A...)-stacking, $\mathrm{MoS}_{2}$ possesses an indirect band gap via $\wedge-\Gamma$, as indicated by the calculation result. This situation is modified for AA (A...)-stacking, where the conduction-band valley at the $\mathrm{K}$ point is aligned with a lower energy than the $\Lambda$-valley by approximately $20 \mathrm{meV}$. We conjectured that C-exciton-gap widening, as observed in the absorption spectra of the AA bilayer, may also result from the relative motion between the conduction-band valleys at the $\mathrm{K}$ and $\wedge$ points. This modification between the conduction-band valleys likely causes the broadening of the indirect band gap, which can be realized with interlayer electronic coupling. ${ }^{3,12,42}$ Additionally, the different alignments between the two conduction bands at the $\mathrm{K}$ and $\wedge$ points also affect the relaxation process at the $\mathrm{K}$ point, leading to an improved direct $\mathrm{PL}$ emission for $\mathrm{AA}(\mathrm{A}$...)-stacking compared to $\mathrm{AB}$ (A...)-stacking.

Furthermore, the theoretical results based on the HSE and PerdewBurke-Ernzerhof approximations agree well with the observed VBS observations. The splitting nature at the $\mathrm{K}$ point is magnified for the $4 \mathrm{~L}-\mathrm{ABAB}$ and $4 \mathrm{~L}-\mathrm{AAAA}$ crystals and is presented in Figure $3 \mathrm{e}$, while related results for $3 \mathrm{~L}$ are presented in the Supplementary Information (Supplementary Figures S9-S11). As expected, for AB(A...)-stacking, the splitting was mainly due to interlayer interactions for an even number of layers, while additional SOC modulated the splitting nature for an odd number of layers. ${ }^{15,16}$ On the other hand, for AA(A...)stacking, the valence-band states at the $\mathrm{K}$ point split into many subbands irrespective of the number of layers. The separation between any two sub-bands is uniform and theoretically estimated as $20 \mathrm{meV}$ for $\mathrm{AA}(\mathrm{A}$...)-stacking, as this stacking remains in the 3R-phase, which does not have a symmetry-inversion center. As a result, SOC becomes dominant. As demonstrated, the splitting is more clearly resolved in the $\mathrm{AA}(\mathrm{A} . .$.$) -stacked crystals than in the \mathrm{AB}(\mathrm{A} . .$.$) -stacked odd layers;$ however, the inversion symmetry is also broken for the latter. Here, more clearly resolved energy states at the $\mathrm{K}$ point of the valence-band maxima for all thicknesses of $\mathrm{AA}(\mathrm{A} . .$.$) -stacked layers can be under-$ stood as strongly spin-polarized states. ${ }^{11,43}$

Experimentally, we cannot resolve each of the spin-polarized states in the PL or absorption spectra, and instead, their average effect must be determined. As previously explained, from the PL and absorption spectra, we observed a smaller gap between two separated VBSs at a $\mathrm{K}$ point for $\mathrm{AA}(\mathrm{A} . .$.$) -stacking than for \mathrm{AB}(\mathrm{A} . .$.$) -stacking. This result is$ caused by the vertical dispersion of the topmost valence bands representing the $\mathrm{A}$ and $\mathrm{B}$ transitions due to strongly polarized spin states. In addition, we note that vertical dispersion of the sub-energy states in the $\mathrm{AA}(\mathrm{A} . .$.$) -stacking due to SOC also slightly influences the$ direct band gap position. The very small band-gap increment $(\sim 5-10 \mathrm{meV})$ beyond $4 \mathrm{~L}$ for $\mathrm{AA}(\mathrm{A} . .$.$) -stacking compared to \mathrm{AB}$ (A...)-stacking reveals the effect of the spin-polarization-induced vertical dispersion of the valence-band edges. It is not surprising that dispersion is more effective for relatively thicker layers because a larger number of sub-bands are induced as a result of splitting. Moreover, we emphasize that our valence-band splitting result is obtained due to the 
a

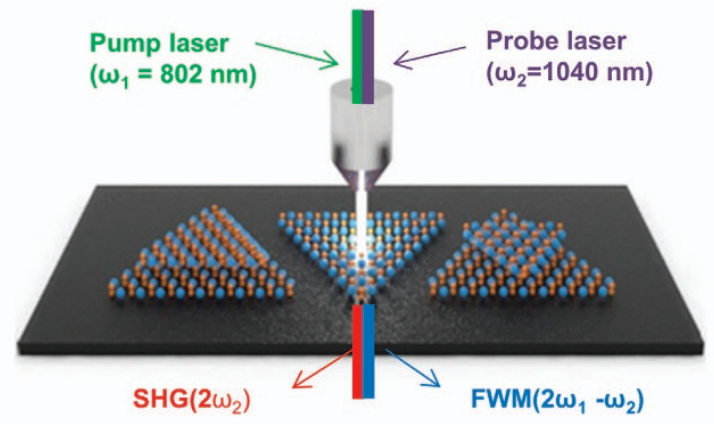

b Laser Power (LP) dependence

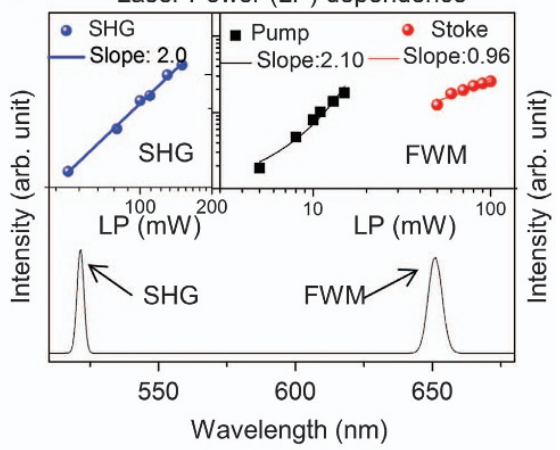

C

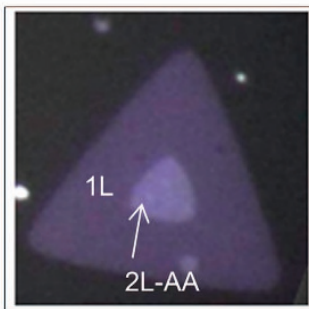

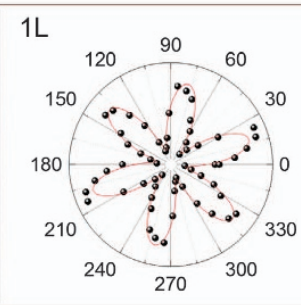

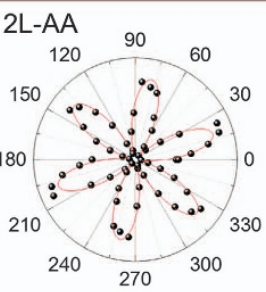

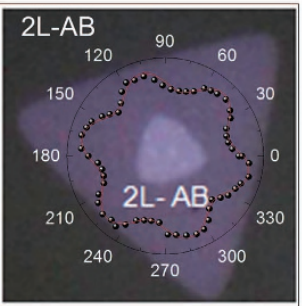

d
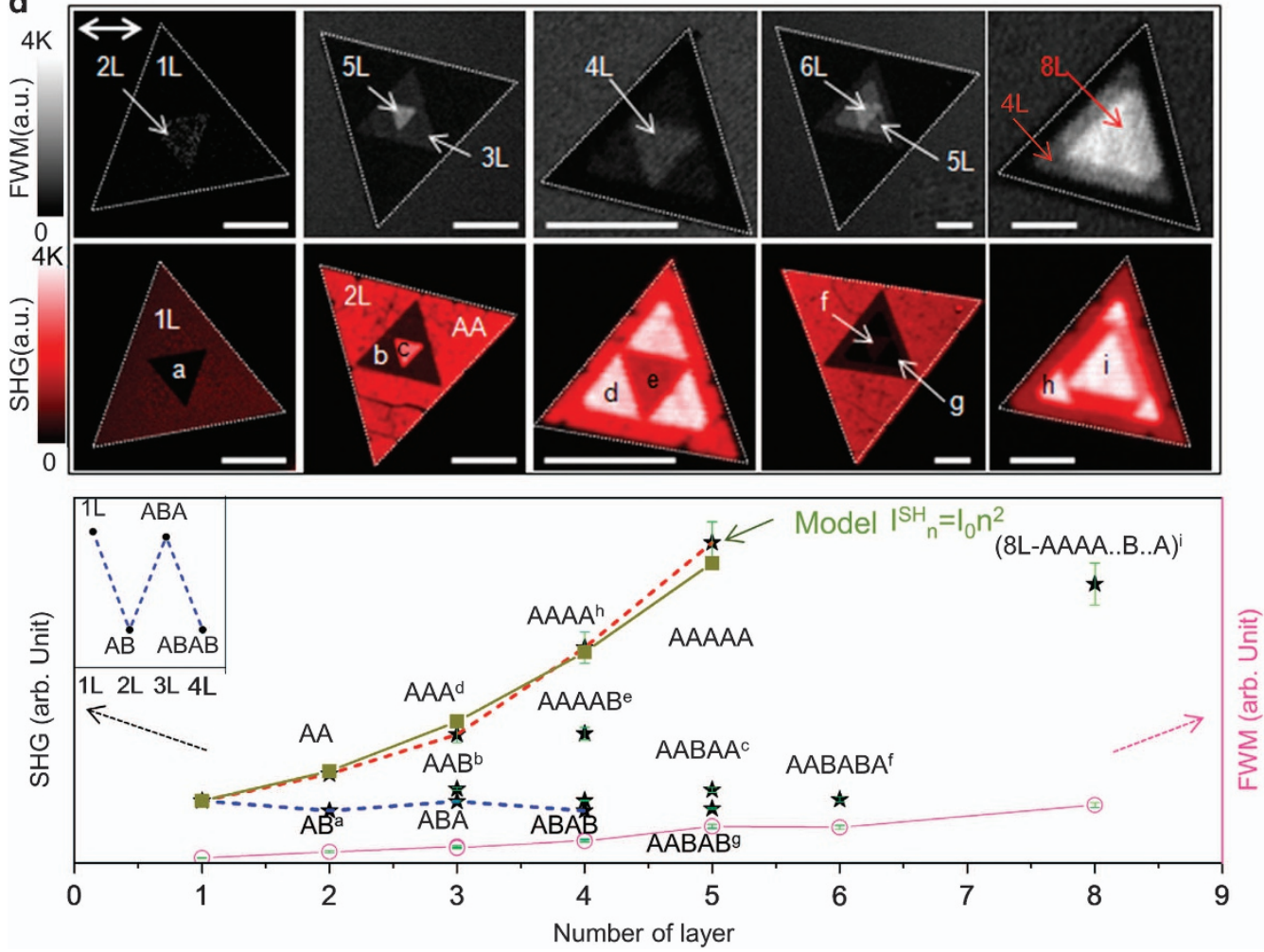

Figure 4 Thickness-dependent FWM and SHG imaging of multilayer $\mathrm{MoS}_{2}$ crystals. (a) A schematic diagram illustrating the interactions between the pumpprobe $(800,1040 \mathrm{~nm})$ laser beams and a stacking-oriented $\mathrm{MoS}_{2}$ atomic-crystal structure, generating second-order SHG and third-order FWM nonlinear optical signals. (b) The spectral evolutions of SHG $(\sim 520 \mathrm{~nm})$ and FWM $(\sim 652 \mathrm{~nm})$ by two excitation beams, together with their laser-power dependencies to assess the nonlinear susceptibilities. (c) Polarization-dependent SHG studies for $1 \mathrm{~L}, 2 \mathrm{~L}-\mathrm{AA}$ and $2 \mathrm{~L}-\mathrm{AB} \mathrm{MoS}_{2}$ (scale bars are $5 \mu \mathrm{m}$ ). (d) The FWM images correlated with the SHG images for different stacking configurations (top panel); the graphs in the bottom panel show the maximum intensity of the SHG for each layer with different stacking orientations, of which the maximum intensity positions were confirmed by rotating the crystal. The inset represents the zoomed SHG intensity variation trend of the ABA...-stacking configuration. The FWM signals from the corresponding crystals (pink ball) are plotted simultaneously (all scale bars are $2 \mu \mathrm{m}$ ). The estimated error bar is provided in the corresponding graph with a green color. The olive color plot represents the results of the model to explain the SH intensity of the AA(A...)-stacking crystals. FWM, four-wave mixing; SHG, second-harmonic signal. 
completely polarized spin states in the $\mathrm{AA}(\mathrm{A} . .$.$) -stacking, which is$ similar to the previously measured valley and spin polarizations of $3 \mathrm{R}-$ phase $\mathrm{MoS}_{2} \cdot{ }^{3,11,43}$ Additionally, 3R-like $\mathrm{MoS}_{2}$ crystals, similar to AA (A...)-stacking, were also accompanied by a suppressed interlayer hopping effect. ${ }^{11}$ All these results indicate that the AA(A...)-stacked crystals of all layer thicknesses exhibited strongly spin-polarized valence-band states.

\section{Stacking-oriented FWM and SHG microscopy}

The structures and orientations of the monolayer to few-layer crystals with different stacking configurations were fully examined through FWM-correlated SHG microscopy. A schematic diagram illustrating the interaction of the pump-probe beam used to emit the nonlinear optical SHG and FWM signals is shown in Figure 4a. The spectral evolutions of SHG $(520 \mathrm{~nm})$ and FWM $(652 \mathrm{~nm})$ by two excitation beams $(800$ and $1040 \mathrm{~nm}$ ) are given in Figure $4 \mathrm{~b}$, where the power dependences of both signals in the inset graphs also suggest that SHG and FWM are second- and third-order nonlinear signals, respectively. Pump and Stokes beams of approximately 1 and $8 \mathrm{~mW}$, respectively, were utilized at the sample for all the SHG/FWM imaging in this work. In principle, the SHG from $\mathrm{MoS}_{2}$ is intense, as the crystalline structure of $\mathrm{MoS}_{2}$ belongs to the non-centrosymmetric point group $\mathrm{D}_{3 \mathrm{~h}} \cdot{ }^{3-5}$ If the input laser and output SHG signal have the same polarization parallel to the monolayer surface, the SHG output is proportional to $\left|\chi_{\mathrm{bbb}}{ }^{(2)}\right|^{2} \cos ^{2} 3 \phi$, where $\chi_{\mathrm{bbb}}{ }^{(2)}$ is the nonlinearsusceptibility element of $\mathrm{MoS}_{2}$, 'b' represents the crystalline axis along the mirror plane, and $\phi$ is the angle of the beam polarization away from the $b$-axis.

We first studied the rotational behaviors of the 1L, 2L-AA and 2L$\mathrm{AB}$ crystals, as shown in the polar plot (Figure $4 \mathrm{c}$ ). The rotational behaviors of $1 \mathrm{~L}$ and $2 \mathrm{~L}-\mathrm{AA}$ were studied from each thickness layer located in the same sample, and the $2 \mathrm{~L}-\mathrm{AB}$ sample was studied in another sample with a different crystal orientation. The incident lasers were linearly polarized and used to excite the samples with the analyzer angle set parallel to the incident beams. All the panoramic SHG images taken with regards to different angles are also provided in Supplementary Figure S12. The six-fold symmetry of the SHG signal with respect to sample rotation was well observed from the respective layers with petals lying along the perpendicular bisectors of each flake, analogous to previously results reported for the $2 \mathrm{H}$ crystal. ${ }^{3,4}$ We note that the $1 \mathrm{~L}, 2 \mathrm{~L}-\mathrm{AA}$ and $3 \mathrm{~L}-\mathrm{AAA}$ crystals demonstrated increased $\mathrm{SH}$ intensity with an increasing thickness due to the cumulative nature of the broken inversion symmetry, whereas the $2 \mathrm{~L}-\mathrm{AB}$ crystal showed feeble SH intensity due to the restored inversion symmetry. Polarization-dependent SHG intensities of the AAA and AAAB stackings are illustrated in Supplementary Figure S12. We estimated the polarization anisotropy $\left\{\left(I_{\max }-I_{\min }\right) /\left(I_{\max }+2 I_{\min }\right)\right\}$ as the degree of SH polarizability for each sample, where $I_{\max }$ and $I_{\min }$ represent the maximum and minimum $\mathrm{SH}$ intensities for the sample rotation, respectively. For the crystals with non-centrosymmetric space groups, that is, $1 \mathrm{~L}, 2 \mathrm{~L}-\mathrm{AA}$ and $3 \mathrm{~L}-\mathrm{AAA}$, the polarization anisotropies were estimated as $70 \%, 80 \%$, and $95 \%$, respectively; this quantity was $10 \%$ for the centrosymmetric $2 \mathrm{H}-\mathrm{AB}$ bilayer. The observed polarization anisotropies of $1 \mathrm{~L}$ and $2 \mathrm{~L}-\mathrm{AB}$ are consistent with the result of the exfoliated sample (Supplementary Figure S13). The 10\% level of remnant $\mathrm{SH}$-polarization anisotropy in the $\mathrm{AB}$ bilayer was assumed to originate from stacking imperfection or substrate-induced imperfection. In contrast, the stronger polarization anisotropies of the AA and AAA crystals are presumably related to physical additions of $\mathrm{SH}-$ generating sources above the monolayers and the increased intensity differences between $I_{\max }$ and $I_{\min }$.
Figure $4 \mathrm{~d}$ presents a simultaneous monitoring of FWM and SHG for the different stacking configurations. The double-sided arrow indicates the directions of the two (pump and Stokes) parallel excitedbeam polarizations. We compared the maximum intensity of the SHG for each layer, of which the position was determined by rotating the crystal. An arbitrary region simultaneously containing various thick crystals with multiple orientation configurations was fully traced via SHG imaging with respect to the sample rotation in Supplementary Figure S14. The FWM signals increased almost linearly with an increasing layer thickness, as previously reported in the literature, while the correlated SHG signal varied according to the stacking configuration. ${ }^{6}$ The $2 \mathrm{H}$-like even-numbered layers with $\mathrm{AB}(\mathrm{A} . .)-$. stacking showed little SHG intensity due to the existence of inversion symmetry and followed the trend indicated by the blue dashed line. The $3 \mathrm{R}$ or AA(A...)-type stacked multilayer crystals presented intense SHG intensities and followed the trend of the red dashed line. Multilayer crystals with mixed stacking orders displayed SHG intensities located between the $2 \mathrm{H}$ and 3R-phases (black stars) depending on the deviation from the stacking orientations of the red/blue dashed lines. Note that all the image correlations between FWM and SHG and their correspondences to the graphs with different stacking orientations are illustrated in the top panel of Figure 4d, where the AA-type bilayer, in contrast to the Bernal-stacked bilayer (AB-type), demonstrates an $\mathrm{SH}$ intensity approximately four times higher than that of 1L. Similarly, the 3L, 4L and 5L AA(A...)-stacked crystals showed drastically enhanced $\mathrm{SH}$ intensities similar to the previously reported results from Zhao et al. ${ }^{44}$ These authors observed a nearly quadratic dependence of the $\mathrm{SH}$ intensity with the layer numbers in a set of exfoliated 3R-like crystals. ${ }^{44}$ To understand our intensity variation with varying layer numbers, we postulate a model based on the electric dipole approximation, which depends on the quadratic variation of the layer numbers in the AA(A...)-stacked crystals. The olive color plot in Figure $4 \mathrm{~d}$ represents the quadratic model, which varies as a function of the layer number. The quadratic model well fits the experimental data and reveals the parallel alignment of the induced dipole in the AA (A...)-stacked crystals contributed strongly to the enhanced $\mathrm{SH}$ intensity. A more detailed description of the model is provided in Supplementary Figures S15 and S16. Additionally, more examples of FWM-correlated SHG images from triangularly shaped crystals with various thicknesses and multiple stacking orders are provided in Figure 5a and Supplementary Figure S17. In general, few stackingoriented signatures are unequivocally identifiable through Raman or PL spectroscopy, except in rigorous studies with TEM or STEM modalities. Here, we found that either AB- or AA-type additions to the bilayer triangles were clearly viewable in the SHG-based image comparison, yet scarcely distinguishable using the optical- or FWMimaging techniques. For example, the SHG signals from two adjacent bilayer crystals are distinctly shown in Figure 5b, indicating the different natures of the inversion symmetry breakage between two bilayers allegedly having FWM intensities with similar levels.

Additionally, a simultaneous FWM and SHG imaging strategy was applied to investigate the various shapes of the CVD-grown $\mathrm{MoS}_{2}$ crystals. Figure $5 \mathrm{c}$ displays the shape-dependent FWM images in the top panel and the corresponding SHG images in the bottom panel. As the first two columns vividly suggest, the perfect hexagonal-shaped monolayer gave a homogeneous SHG contrast generated by the single crystal, whereas the 'domains' of the imperfect hexagonal or polygonal samples were selectively visualized by SHG. In other words, neither FWM nor optical images (top panel) could discriminate meaningful contrast from either the single or polycrystalline monolayer, while the SHG images successfully resolved the polycrystalline domain within a 


\section{a}

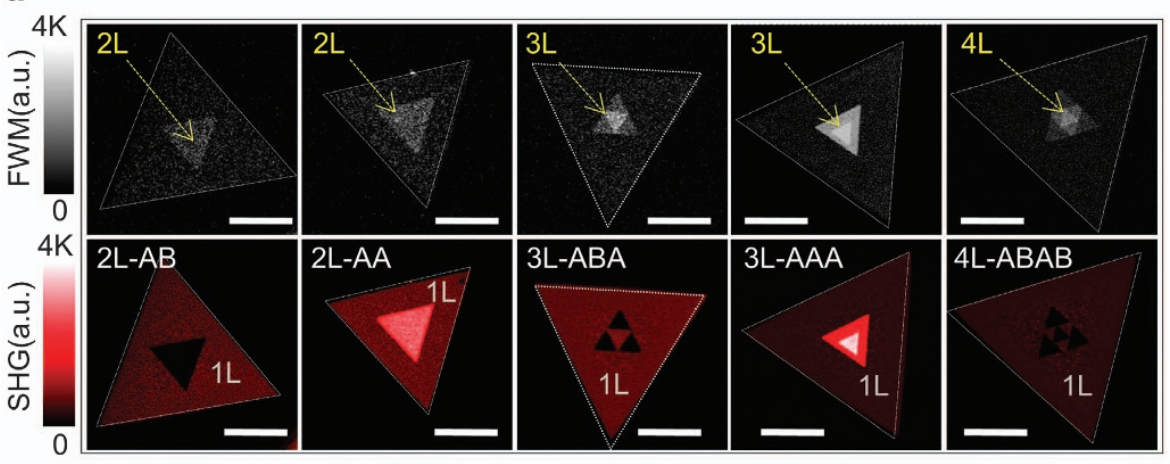

b
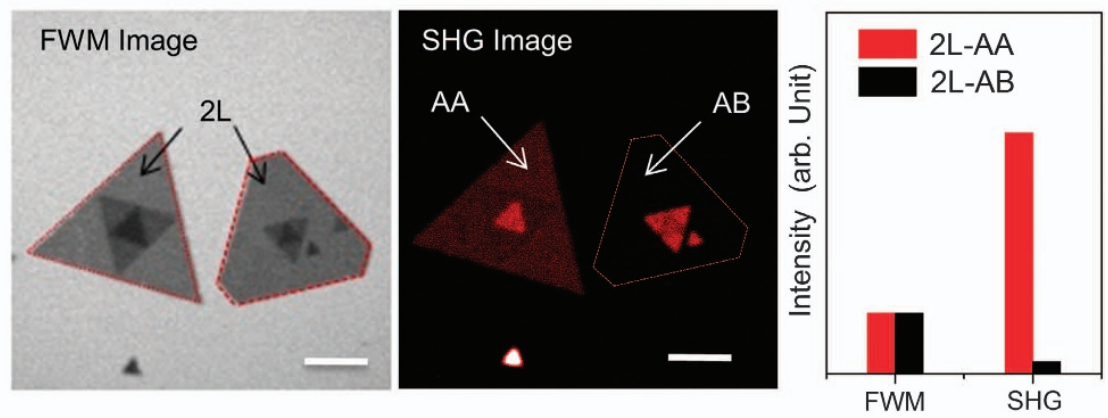

C
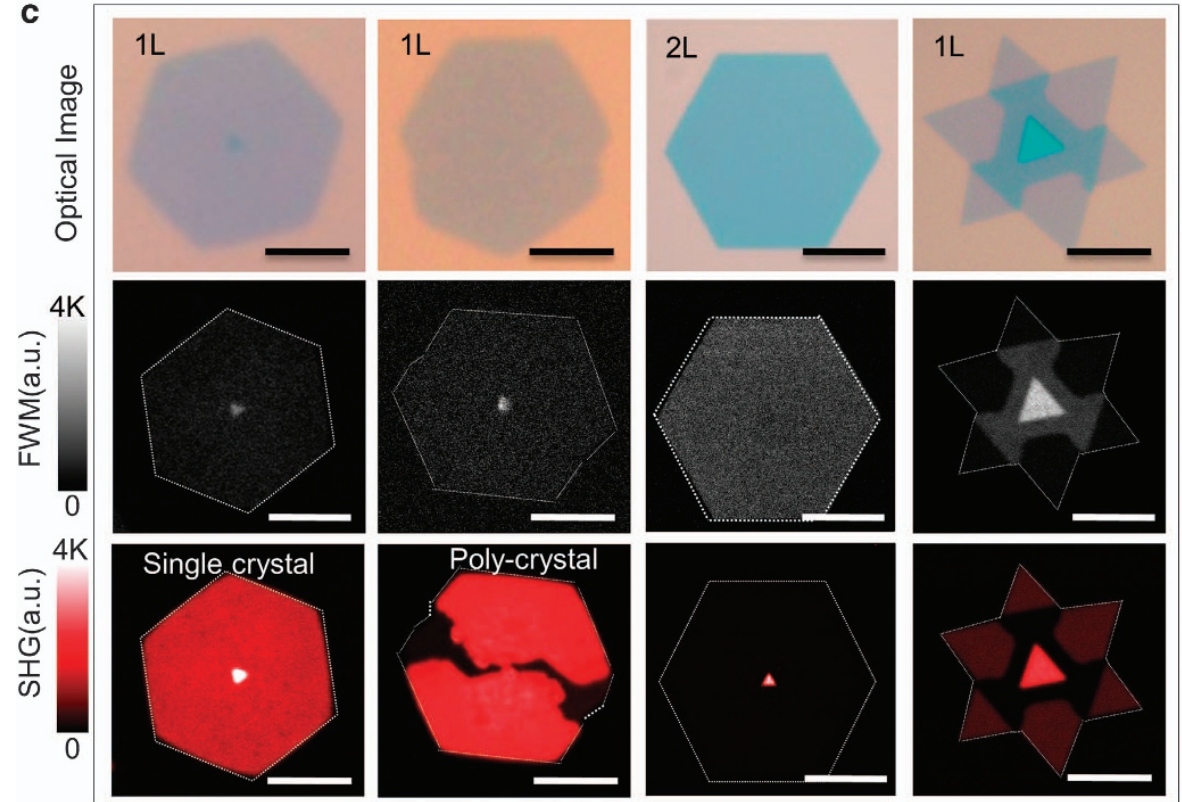

Figure 5 Shape- and stacking-orientation-dependent FWM and SHG imaging of multilayer $\mathrm{MoS}_{2}$ crystals. (a) Various types of layer stackings, starting either from monolayer or bilayer $\mathrm{MoS}_{2}$ crystals, resolved by collaborative FWM and SHG imaging. (b) Intensity comparison of the FWM and SHG images from two adjacent twin bilayer crystals having AA- and AB-stacking orientations. The AA-type stacked bilayer shows intense SHG compared to the AB-type bilayer, whereas the FWM images are identical regardless of the stacking order. (c) The optical, FWM and SHG images for differently shaped single-crystalline and polycrystalline $\mathrm{MoS}_{2}$ systems. Scale bars correspond to $2 \mu \mathrm{m}$. FWM, four-wave mixing; SHG, second-harmonic signal; MoS 2 , molybdenum disulfide.

flake specimen created by the azimuthal-angle dependence of the SHG signal with respect to the incident light polarization. An extended view of the region simultaneously containing poly- and single-crystalline flakes is presented in Supplementary Figure S14.

Moreover, all the triangles stacked on the 1L-hexagonal crystal showed enhanced SHG irrespective of their stacking orientations, as illustrated in Supplementary Figure S18b. The SHG intensity ratio between the 1L-hexagon and hexagon/triangle bilayer was almost identical to that between the 1L-triangle and triangle/triangle bilayer. It is possible that the stacking orientation between two heterogeneously shaped crystals favors a staggered-lattice structure between the layers. A hexagonal bilayer is formed as a $2 \mathrm{H}$-like phase with an inversion symmetry center, as indicated by the lack of SHG contrast. Interestingly, we can also visualize a star-shaped crystal via not only the thickness using FWM but also the stacking orientation using SHG; this crystal is clearly distinct from previously reported results that 
mentioned only polycrystalline star-shaped monolayers. ${ }^{4}$ Here, a single-crystalline star-shaped crystal with six arms was observable, where added layers were grown in the central region. The region of the black SHG contrast inside the 1L-star-shaped flake was created by the reversely oriented growth of the second layer. The bright central SH emission was allowed by the addition of more layers with stacking orientations identical to that of the first layer, where stacked layers in the star-shaped crystal could also be identified in the optical and FWM images. We believe that our raster-scanning SHG technique is helpful to quickly resolve the polarization-induced phase contrasts of various multilayered 2D-transition-metal dichalcogenide films, thereby providing a new way to design optically triggered CVD-based $2 \mathrm{D}$ optoelectronics.

In summary, we developed a method to synthesize multilayer $\mathrm{MoS}_{2}$ crystals with different stacking orientations by APCVD using $\mathrm{NaCl}$ as a growth promoter. $\mathrm{NaCl}$ was found to play a vital role in the formation of Mo-containing nucleation sites at a lower temperature, and $\mathrm{Na}$ ions helped weaken the interlayer adhesion, contributing to an increase in the growth rate for highly stacked $\mathrm{MoS}_{2}$ crystals with different orientations. The growth temperature dependence upon the stacking order and shape of multi-stacked crystals was studied. We revealed the stacking-orientation dependence of the crystal symmetry, phase, and associated electron-phonon coupling in the electronic band structure using optical investigations based on Raman spectroscopy, PL spectroscopy and a nonlinear technique of FWM-correlated SHG imaging. The Raman frequencies induced by the $\mathrm{AA}(\mathrm{A} . .$.$) -stacking orientation$ clearly stiffened the $E_{2 g}{ }^{1}$ and $A_{1 g}$ phonon vibrations for layer thicknesses greater than $3 \mathrm{~L}$ as compared to those induced by the $\mathrm{AB}$ (A...)-stacking configuration, indicating enhanced interlayer interactions. The stacking dependence of the phonon vibrations can be used as a robust and convenient means of diagnosing interlayer interactions and structural changes. The experimental and theoretical study based on excitonic properties suggests that the stacking orientation is the key to engineering a fundamental band structure and improved PL emission in multilayer crystals with $\mathrm{AA}(\mathrm{A} . .$.$) configurations. More-$ over, highly spin-polarized states were observed in $\mathrm{AA}(\mathrm{A} . .$.$) -stacked$ crystals of all thicknesses, in contrast to naturally stacked $\mathrm{AB}(\mathrm{A} . .$. crystals, in which only odd numbers of multilayers exhibited spin polarization. Furthermore, the nonlinear FWM-correlated SHGimage-based study showed that the $\mathrm{AA}(\mathrm{A} . .$.$) -stacked-multilayer$ $\mathrm{MoS}_{2}$ exhibited stronger $\mathrm{SH}$ intensities for crystals of all thicknesses than those of $\mathrm{AB}(\mathrm{A} . .$.$) -stacked crystals, thereby confirming that the$ broken structural symmetry in $\mathrm{AA}\left(\mathrm{A}\right.$...)-stacked-multilayer $\mathrm{MoS}_{2}$ manifested stronger SOC-induced band splitting, as supported by first-principles calculations. FWM is stacking-orientation independent and exhibited a linear dependence on the layer thickness. We carefully inspected the FWM and SHG intensities and confirmed the structural symmetry by detecting an optically invisible stacking orientation in a multi-stacked CVD crystal with $2 \mathrm{H}$ and $3 \mathrm{R}$ phases in the Brillouin zone. Our study based on crystals with different shapes and stacking configurations provides a new avenue for developing future optoelectronic devices.

\section{CONFLICT OF INTEREST}

The authors declare no conflict of interest.

\section{ACKNOWLEDGEMENTS}

This work was supported by the National Research Foundation of Korea (NRF-2015R1A3A2066337) and the DGIST Basic Research Program (17BT-01) funded by the Ministry of Science and ICT of Korea.
Author contributions: J-HA planned and supervised the project. HK co-supervised the project. SMS performed the CVD growth of $\mathrm{MoS}_{2}$. KPD and SMS carried out the PL, Raman and absorption characterizations. XC performed the AFM imaging. KPD and HK performed the nonlinear optical characterization and analyzed the optical data. WSY and JDL performed the DFT calculations. KPD, SMS, XC, HK and J-HA wrote the manuscript.

\section{PUBLISHER'S NOTE}

Springer Nature remains neutral with regard to jurisdictional claims in published maps and institutional affiliations.

1 Mak, K. F., Lee, C., Hone, J., Shan, J. \& Heinz, T. F. Atomically thin $\mathrm{MoS}_{2}$ : a new direct-gap semiconductor. Phys. Rev. Lett. 105, 136805 (2010).

2 Dhakal, K. P., Duong, D. L., Lee, J., Nam, H., Kim, M., Kan, M., Lee, Y. H. \& Kim, J. Confocal absorption spectral imaging of $\mathrm{MoS}_{2}$ : optical transitions depending on the atomic thickness of intrinsic and chemically doped $\mathrm{MoS}_{2}$. Nanoscale 6 , 13028-13035 (2014).

3 Jiang, T., Liu, H., Huang, D., Zhang, S., Li, Y., Gong, X., Shen, Y. R., Liu, W. T. \& Wu, S. Valley and band structure engineering of folded MoS2 bilayers. Nat. Nanotechnol. $\mathbf{9}$, 825-829 (2014).

4 Hsu, W. T., Zhao, Z. A., Li, L. J., Chen, C. H., Chiu, M. H., Chang, P. S., Chou, Y.C. \& Chang, W.H. Second harmonic generation from artificially stacked transition metal dichalcogenide twisted bilayers. ACS Nano 8, 2951-2958 (2014).

5 Li, Y., Rao, Y., Mak, K. F., You, Y., Wang, S., Dean, C. R. \& Heinz, T. F. Probing symmetry properties of few-layer $\mathrm{MoS}_{2}$ and h-BN by optical second-harmonic generation. Nano Lett. 13, 3329-3333 (2013).

6 Li, D., Xiong, W., Jiang, L., Xiao, Z., Golgir, H. R., Wang, M., Huang, X., Zhou, Y., Lin, Z., Song, J., Ducharme, S., Jiang, L., Silvain, J. F. \& Lu, Y. Multimodal nonlinear optical imaging of $\mathrm{MoS}_{2}$ and $\mathrm{MoS}_{2}$-based van der Waals heterostructures. ACS Nano 10, 3766-3775 (2016)

7 Park, S., Kim, M. S., Kim, H., Lee, J., Han, G. H., Jung, J. \& Kim, J. Spectroscopic visualization of grain boundaries of monolayer molybdenum disulfide by stacking bilayers. ACS Nano 9, 11042-11048 (2015).

8 Kozawa, D., Kumar, R., Carvalho, A., Kumar Amara, K., Zhao, W., Wang, S., Toh, M., Ribeiro, R. M., Castro Neto, A. H., Matsuda, K. \& Eda, G. Photocarrier relaxation pathway in two-dimensional semiconducting transition metal dichalcogenides. Nat. Commun. 5, 1-7 (2014).

9 Chen, X., Park, Y. J., Das, T., Jang, H., Lee, J. B. \& Ahn, J. H. Lithography-free plasmainduced patterned growth of $\mathrm{MoS}_{2}$ and its heterojunction with graphene. Nanoscale 8 , 15181-15188 (2016).

10 Zheng, J., Yan, X., Lu, Z., Quu, H., Xu, G., Zhou, X., Wang, P., Pan, X., Liu, K. \& Jiao, L. High-mobility multilayered $\mathrm{MoS}_{2}$ flakes with low contact resistance grown by chemical vapor deposition. Adv. Mater. 29, 1604540 (2017).

11 Yan, J., Xia, J., Wang, X., Liu, L., Kuo, J. L., Tay, B. K., Chen, S., Zhou, W., Liu, Z. \& Shen, Z. X. Stacking-dependent interlayer coupling in trilayer $\mathrm{MoS}_{2}$ with broken inversion symmetry. Nano Lett. 15, 8155-8161 (2015).

12 Xia, M., Li, B., Yin, K., Capellini, G., Niu, G., Gong, Y., Zhou, W., Ajayan, P. M. \& Xie, Y. $\mathrm{H}$. Spectroscopic signatures of $A A^{\prime}$ and $A B$ stacking of chemical vapor deposited bilayer $\mathrm{MoS}_{2}$. ACS Nano 9, 12246-12254 (2015).

13 Yan, A., Chen, W., Ophus, C., Ciston, J., Lin, Y., Persson, K. \& Zettl, A. Identifying different stacking sequences in few-layer CVD-grown $\mathrm{MoS}_{2}$ by low-energy atomicresolution scanning transmission electron microscopy. Phys. Rev. B 93, 041420 (2016).

14 Gong, Y., Ye, G., Lei, S., Shi, G., He, Y., Lin, J., Zhang, X., Vajtai, R., Pantelides, S. T. \& Zhou, W. Synthesis of millimeter-scale transition metal dichalcogenides single crystals. Adv. Funct. Mater. 26, 2009-2015 (2016).

15 Li, S., Wang, S., Tang, D. M., Zhao, W., Xu, H., Chu, L., Bando, Y., Golberg, D. \& Eda, G. Halide-assisted atmospheric pressure growth of large $\mathrm{WS}_{2}$ and $\mathrm{WS}_{2}$ monolayer crystals. Appl. Mater. Today 1, 60-66 (2015).

16 Latzke, D. W., Zhang, W., Suslu, A., Chang, T. R., Lin, H., Jeng, H. T., Tongay, S., Wu, J., Bansil, A. \& Lanzara, A. Electronic structure, spin-orbit coupling, and interlayer interaction in bulk $\mathrm{MoS}_{2}$ and $\mathrm{WS}_{2}$. Phys. Rev. B 91, 235202 (2015).

17 Jin, W., Yeh, P. C., Zaki, N., Zhang, D., Sadowski, J. T., Al-Mahboob, A., van der Zande, A. M., Chenet, D. A., Dadap, J. I., Herman, I. P., Sutter, P., Hone, J. \& Osgood, R. M. Jr. Direct measurement of the thickness-dependent electronic band structure of $\mathrm{MoS}_{2}$ using angle-resolved photoemission spectroscopy. Phys. Rev. Lett. 111, 106801 (2013).

18 Lee, J-U., Kim, K., Han, S., Ryu, G. H., Lee, Z. \& Cheong, H. Raman signatures of polytypism in molybdenum disulfide. ACS Nano 10, 1948 (2016).

19 Blöchl, P. E. Projector augmented-wave method. Phys. Rev. B 50, 17953-17979 (1994).

20 Kresse, G. \& Joubert, D. From ultrasoft pseudopotentials to the projector augmentedwave method. Phys. Rev. B 59, 1758-1775 (1999).

21 Kresse, G. \& Hafner, J. Ab initio molecular dynamics for liquid metals. Phys. Rev. B 47, 558-561 (1993).

22 Perdew, J. P., Burke, K. \& Ernzerhof, M. Generalized gradient approximation made simple. Phys. Rev. Lett. 77, 3865-3868 (1996). 
23 Klimeš, J., Bowler, D. R. \& Michaelides, A. Chemical accuracy for the Van der Waals density functional. J. Phys. Condens. Matter. 22, 022201 (2010).

24 Klimeš, J., Bowler, D. R. \& Michaelides, A. Van der Waals density functionals applied to solids. Phys. Rev. B 83, 195131 (2011).

25 Heyd, J., Scuseria, G. E. \& Ernzerhof, M. Hybrid functionals based on a screened Coulomb potential. J. Chem. Phys. 118, 8207-8215 (2003)

26 Lee, D., Jeong, D.-E., Son, H. G., Yamaoka, Y., Kim, H., Seo, K., Khan, A. A., Roh, T.-Y., Moon, D. W., Lee, Y. \& Lee, S.-J.V. SREBP and MDT-15 protect C. elegans from glucose-induced accelerated aging by preventing accumulation of saturated fat Genes \& Dev 29, 2490-2503 (2015).

27 Kim, H., Kim, D.-Y., Joo, K.-I., Kim, J.-H., Jeong, S.M., Lee, E. S., Hahm, J.-H., Kim, K. \& Moon, D.W. Coherent Raman imaging of live muscle sarcomeres assisted by SFG microscopy. Sci. Rep. 7, 9211 (2017).

28 Johnson, D. A., Levy, J. H., Taylor, J. C., Waugh, A. B. \& Brough, J. Purification of molybdenum: volatilisation processes using $\mathrm{MoO}_{3}$. Polyhedron 1, 479-482 (1982).

29 Wang, S., Rong, Y., Fan, Y., Pacios, M., Bhaskaran, H., He, K. \& Warner, J. H. Shape evolution of monolayer $\mathrm{MoS}_{2}$ crystals grown by chemical vapor deposition. Chem. Mater. 26, 6371-6379 (2014).

30 Li, S. F., Zhao, X. J., Xu, X. S., Gao, Y. \& Zhang, Z.Y. Stacking principle and magic sizes of transition metal nanoclusters based on generalized wulff construction. Phys. Rev. Lett. 111, 115501 (2013).

31 Cao, D., Shen, T., Liang, P., Chen, X. S. \& Shu, H. B. Role of chemical potential in flake shape and edge properties of monolayer $\mathrm{MoS}_{2}$. J. Phys. Chem. C 119, 4294-4301 (2015).

32 Xie, Y., Wang, Z., Zhan, Y., Zhang, P., Wu, R., Jiang, T., Wu, S., Wang, H., Zhao, Y., Nan, T. \& Ma, X. Controllable growth of monolayer $\mathrm{MoS}_{2}$ by chemical vapor deposition via close $\mathrm{MoO}_{2}$ precursor for electrical and optical applications. Nanotechnology 28 084001 (2017)

33 Lauritsen, J. V., Kibsgaard, J., Helveg, S., Topsøe, H., Clausen, B. S., Laegsgaard, E. \& Besenbacher, F. Size-dependent structure of MoS2 nanocrystals. Nat. Nanotechnol. 2 53-58 (2007).

34 Rajan, A. G., Warner, J. H., Blankschtein, D. \& Strano, M. S. Generalized mechanistic model for the chemical vapor deposition of 2D transition metal dichalcogenide monolayers. ACS Nano 10, 4330-4344 (2016).

35 Lee, C., Yan, H., Brus, L. E., Heinz, T. F., Hone, J. \& Ryu, S. Anomalous lattice vibrations of single- and few-layer MoS 2 . ACS Nano 4, 2695-2700 (2010).

36 Jeong, H. Y., Jin, Y., Yun, S. J., Zhao, J., Baik, J., Keum, D. H., Lee, H. S. \& Lee, Y. H. Heterogeneous defect domains in single-crystalline hexagonal WS 2 . Adv. Mater. 29, 1605043 (2017).

37 Liu, Z., Amani, M., Najmaei, S., Xu, Q., Zou, X., Zhou, W., Yu, T., Qiu, C., Birdwell, A. G., Crowne, F. J., Vajtai, R., Yakobson, B. I., Xia, Z., Dubey, M., Ajayan, P. M. \& Lou, J.
Strain and structure heterogeneity in $\mathrm{MoS}_{2}$ atomic layers grown by chemical vapour deposition. Nat. Commun. 5, 1-9 (2014).

$38 \mathrm{He}$, K., Poole, C., Mak, K. F. \& Shan, J. Experimental demonstration of continuous electronic structure tuning via strain in atomically thin $\mathrm{MoS}_{2}$. Nano Lett. 13, 2931-2936 (2013).

39 Liang, L. \& Meunier, V. First-principles Raman spectra of $\mathrm{MoS}_{2}, \mathrm{WS}_{2}$ and their heterostructures. Nanoscale 6, 5394-5401 (2014).

40 Amani, M., Burke, R. A., Ji, X., Zhao, P., Lien, D. H., Taheri, P., Ahn, G. H., Kirya, D. Ager, J. W. 3rd, Yablonovitch, E., Kong, J., Dubey, M. \& Javey, A. High luminescence efficiency in $\mathrm{MoS}_{2}$ grown by chemical vapor deposition. ACS Nano 10, 6535-6541 (2016).

41 Amani, M., Lien, D. H., Kiriya, D., Xiao, J., Azcatl, A., Noh, J., Madhvapathy, S. R., Addou, R., Santosh, K. C., Dubey, M., Cho, K., Wallace, R. M., Lee, S. C., He, J. H., Ager, J. W., Zhang, X., Yablonovitch, E. \& Javey, A. Near-unity photoluminescence quantum yield in $\mathrm{MoS}_{2}$. Science 350, 1065-1068 (2015).

42 Liu, K., Zhang, L., Cao, T., Jin, C., Qiu, D., Zhou, Q., Zettl, A., Yang, P., Louie, S. G. \& Wang, F. Evolution of interlayer coupling in twisted molybdenum disulfide bilayers. Nat. Commun. 5, 4966 (2014).

43 Suzuki, R., Sakano, M., Zhang, Y. J., Akashi, R., Morikawa, D., Harasawa, A., Yaji, K., Kuroda, K., Miyamoto, K., Okuda, T., Ishizaka, K., Arita, R. \& Iwasa, Y. Valleydependent spin polarization in bulk $\mathrm{MoS}_{2}$ with broken inversion symmetry. Nat Nanotech. 9, 611-617 (2014).

44 Zhao, M., Ye, Z., Suzuki, R., Ye, Y., Zhu, H., Xiao, J., Wang, Y., Iwasa, Y. \& Zhang, X Atomically phase-matched second-harmonic generation in a 2D crystal. Light: Sci. Appl. 5, e16131 (2016).

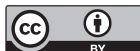

This work is licensed under a Creative Commons Attribution 4.0 International License. The images or other third party material in this article are included in the article's Creative Commons license, unless indicated otherwise in the credit line; if the material is not included under the Creative Commons license, users will need to obtain permission from the license holder to reproduce the material. To view a copy of this license, visit http:// creativecommons.org/licenses/by/4.0/

(C) The Author(s) 2018

Supplementary Information accompanies the paper on the NPG Asia Materials website (http://www.nature.com/am) 